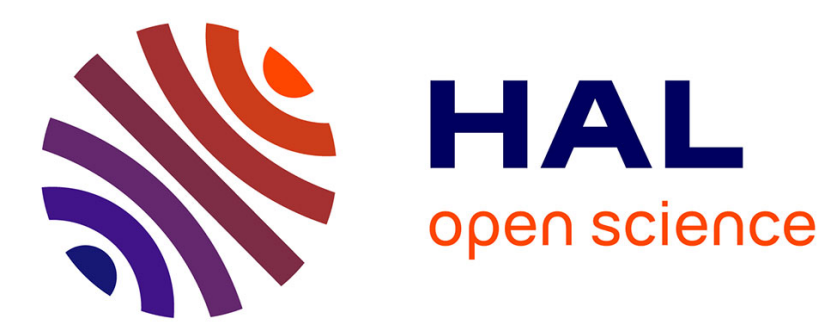

\title{
Multiphase Flow Modeling via Hamilton's Principle
} Sergey Gavrilyuk

\section{To cite this version:}

Sergey Gavrilyuk. Multiphase Flow Modeling via Hamilton's Principle. 2011. hal-00601779

\section{HAL Id: hal-00601779 \\ https://hal.science/hal-00601779}

Preprint submitted on 20 Jun 2011

HAL is a multi-disciplinary open access archive for the deposit and dissemination of scientific research documents, whether they are published or not. The documents may come from teaching and research institutions in France or abroad, or from public or private research centers.
L'archive ouverte pluridisciplinaire HAL, est destinée au dépôt et à la diffusion de documents scientifiques de niveau recherche, publiés ou non, émanant des établissements d'enseignement et de recherche français ou étrangers, des laboratoires publics ou privés. 


\title{
Multiphase Flow Modeling via Hamilton's Principle
}

\author{
Sergey Gavrilyuk * \\ * Aix-Marseille University, CNRS UMR 6595, IUSTI, Marseille, France \\ $\ddagger$ CISM, International Center for Mechanical Sciences, Udine, Italy
}

\begin{abstract}
We present here a variational approach to derivation of multiphase flow models. Two basic ingredients of this method are as follows. First, a conservative part of the model is derived based on the Hamilton principle of stationary action. Second, phenomenological dissipative terms are added which are compatible with the entropy inequality. The variational technique is shown up, and mathematical models (classical and non-classical) describing fluid-fluid and fluid-solid mixtures and interfaces are derived.
\end{abstract}

\section{Introduction}

For any vectors $\mathbf{a}, \mathbf{b}$ we shall use the notations $\mathbf{a} \cdot \mathbf{b}$ for their scalar product (the line vector (covector) is multiplied by the column vector) and $\mathbf{a} \otimes \mathbf{b}$ for their tensor product (the column vector is "multiplied" by the line vector). Linear transformations of vectors into vectors (second order tensors) are denoted by uppercase letters. The divergence of a linear transformation $A$ is the covector $\operatorname{div} A$ such that, for any constant vector a

$$
\operatorname{div}(A) \cdot \mathbf{a}=\operatorname{div}(A \mathbf{a}) .
$$

In particular, for any vector field $\mathbf{v}$

$$
\begin{gathered}
\operatorname{div}(A \mathbf{v})=\operatorname{div} A \cdot \mathbf{v}+\operatorname{tr}\left(A \frac{\partial \mathbf{v}}{\partial \mathbf{x}}\right) \\
\operatorname{div}(\mathbf{u} \otimes \mathbf{v})=\mathbf{v} \operatorname{div} \mathbf{u}+\frac{\partial \mathbf{v}}{\partial \mathbf{x}} \mathbf{u}
\end{gathered}
$$

In the last formula we have been identified the line vectors and the column vectors. The identical transformation is denoted by $I$, and the gradient operator by $\nabla$. The elements of a linear transformation $A$ in a given base 
$\mathbf{e}_{j}, j=1,2,3$ are denoted be $A_{j}^{i}: A \mathbf{e}_{j}=A_{j}^{i} \mathbf{e}_{i}$ where $i$ means lines and $j$ columns. The repeated Latin indices imply summation. In particular,

$$
(\mathbf{a} \otimes \mathbf{b})_{j}^{i}=a^{i} b_{j} .
$$

If $f(A)$ is a scalar function of $A$, the matrix of $\frac{\partial f}{\partial A}$ is defined by the formula

$$
\left(\frac{\partial f}{\partial A}\right)_{i}^{j}=\frac{\partial f}{\partial A_{j}^{i}}
$$

The definition of the divergence of a linear transformation used in France is opposite to the one used in USA where the divergence of $A$ is defined as follows

$$
\operatorname{div}(A) \cdot \mathbf{a}=\operatorname{div}\left(A^{T} \mathbf{a}\right)
$$

for any constant vector a. Once an appropriate definition has been selected, it should remain unchanged during calculations.

\section{Variations}

The results of this Section are well known and can be partially found, for example, in the book by Berdichevsky (2009).

Let $\mathcal{D}(t)$ be a volume of the physical space occupied by a fluid at time $t$. A particle is labeled by its initial position $\mathbf{X}$ in the reference space $\mathcal{D}\left(t_{0}\right)$, the motion of the continuum is defined as a diffeomorphism from $\mathcal{D}\left(t_{0}\right)$ into $\mathcal{D}(t)$ :

$$
\mathbf{x}=\varphi_{t}(\mathbf{X})
$$

The velocity $\mathbf{u}$ and the deformation gradient $F$ are defined by

$$
\mathbf{v}=\frac{\partial \varphi_{t}(\mathbf{X})}{\partial t}, \quad F=\frac{\partial \mathbf{x}}{\partial \mathbf{X}}=\frac{\partial \varphi_{t}(\mathbf{X})}{\partial \mathbf{X}}
$$

Let

$$
\mathbf{x}=\mathbf{\Phi}_{t}(\mathbf{X}, \varepsilon)
$$

be a one-parameter family of virtual motions of the medium such that

$$
\left.\boldsymbol{\Phi}_{t}(\mathbf{X}, \varepsilon)\right|_{\varepsilon=0}=\boldsymbol{\varphi}_{t}(\mathbf{X}) .
$$

Here $\varepsilon$ is a scalar defined in the vicinity of zero. We define the virtual displacement $\delta \mathbf{x}(t, \mathbf{X})$ associated with the virtual motion :

$$
\delta \mathbf{x}(t, \mathbf{X})=\left.\frac{\partial \boldsymbol{\Phi}_{t}(\mathbf{X}, \varepsilon)}{\partial \varepsilon}\right|_{\varepsilon=0} .
$$


Let us consider any quantity $f$ defined in the Eulerian coordinates (or in the Lagrangian coordinates)

$$
(t, \mathbf{x}) \longrightarrow f(t, \mathbf{x})
$$

or

$$
(t, \mathbf{X}) \rightarrow f(t, \mathbf{X})
$$

We abuse the notation by using the same letter $f$ for completely different functions, because the last one is obtained from the first one by replacing $\mathbf{x}=\varphi_{t}(\mathbf{X})$. To avoid a possible confusion, we will often indicate the arguments of these functions. The corresponding virtual families of $f$ in the Eulerian coordinates will be denoted by $\hat{f}(t, \mathbf{x}, \varepsilon)$ and $\tilde{f}(t, \mathbf{X}, \varepsilon)$. The relation between them is as follows:

$$
\tilde{f}(t, \mathbf{X}, \varepsilon)=\hat{f}\left(t, \Phi_{t}(\mathbf{X}, \varepsilon), \varepsilon\right)
$$

Let us define the Eulerian variations of $f$

$$
\hat{\delta} f(t, \mathbf{x})=\left.\frac{\partial \hat{f}}{\partial \varepsilon}\right|_{\varepsilon=0}
$$

and the Lagrangian variation :

$$
\tilde{\delta} f(t, \mathbf{X})=\left.\frac{\partial \tilde{f}}{\partial \varepsilon}\right|_{\varepsilon=0}
$$

Differentiating (2) with respect to $\varepsilon$ we obtain the following relation between two types of variations :

$$
\tilde{\delta} f=\hat{\delta} f+\frac{\partial f}{\partial \mathbf{x}} \delta \mathbf{x}=\hat{\delta} f+\nabla f \cdot \delta \mathbf{x}
$$

\section{Variation of density}

The density $\rho(t, \mathbf{X})$ is determined by

$$
\rho \operatorname{det} F=\rho_{0}(\mathbf{X}),
$$

where $\rho_{0}(\mathbf{X})$ is the reference density.

\section{Lemma}

$$
\tilde{\delta} F=\frac{\partial \delta \mathbf{x}}{\partial \mathbf{X}}, \quad \tilde{\delta}(\operatorname{det} F)=\operatorname{det} F \operatorname{tr}\left(F^{-1} \tilde{\delta} F\right)=\operatorname{det} F \operatorname{div}(\delta \mathbf{x})
$$


Here the variation $\delta \mathbf{x}$ defined by (1) is considered as a function of the Eulerian coordinates. In the following, all operations from the vector analysis (div, $\nabla, \ldots$ ) will be applied only to functions of the Eulerian coordinates.

Proof

By definition,

$$
\tilde{F}(t, \mathbf{X}, \varepsilon)=\frac{\partial \Phi_{t}(\mathbf{X}, \varepsilon)}{\partial \mathbf{X}} .
$$

Differentiating it ones with respect to $\varepsilon$ we obtain the first formula. The proof of the second formula we present below is due to P. Casal. Let $\mathbf{w}_{1}$, $\mathbf{w}_{2}, \ldots, \mathbf{w}_{n}$ be $n$ constant vectors. We denote by $\operatorname{det}\left(\mathbf{w}_{1}, \mathbf{w}_{2}, \ldots, \mathbf{w}_{n}\right)$ the determinant of the matrix having columns $\mathbf{w}_{1}, \mathbf{w}_{2}, \ldots, \mathbf{w}_{n}$. For any linear transformation $A$ its determinant and its trace satisfy the following relations:

$$
\begin{array}{r}
\operatorname{det}\left(A \mathbf{w}_{1}, A \mathbf{w}_{2}, \ldots, A \mathbf{w}_{n}\right)=\operatorname{det} A \operatorname{det}\left(\mathbf{w}_{1}, \mathbf{w}_{2}, \ldots, \mathbf{w}_{n}\right), \\
\operatorname{tr}(A) \operatorname{det}\left(\mathbf{w}_{1}, \mathbf{w}_{2}, \ldots, \mathbf{w}_{n}\right) \\
=\operatorname{det}\left(A \mathbf{w}_{1}, \mathbf{w}_{2}, \ldots, \mathbf{w}_{n}\right)+\ldots+\operatorname{det}\left(\mathbf{w}_{1}, \mathbf{w}_{2}, \ldots, A \mathbf{w}_{n}\right) .
\end{array}
$$

Hence

$$
\begin{aligned}
d(\operatorname{det} A) \operatorname{det}\left(\mathbf{w}_{1}, \mathbf{w}_{2}, \ldots, \mathbf{w}_{n}\right)=\operatorname{det}\left(d A \mathbf{w}_{1}, A \mathbf{w}_{2}, \ldots, A \mathbf{w}_{n}\right) & +\ldots+\operatorname{det}\left(A \mathbf{w}_{1}, A \mathbf{w}_{2}, \ldots, d A \mathbf{w}_{n}\right) \\
& \left.+\ldots \mathbf{w}_{n}\right)+\ldots \\
= & \operatorname{det}\left(d A A^{-1} A \mathbf{w}_{1}, A \mathbf{w}_{2}, \ldots, A \mathbf{w}_{n}\right) \\
& \quad+\operatorname{det}\left(A \mathbf{w}_{1}, A \mathbf{w}_{2}, \ldots, d A A^{-1} A \mathbf{w}_{n}\right) \\
= & \operatorname{tr}\left(d A A^{-1}\right) \operatorname{det}\left(A \mathbf{w}_{1}, A \mathbf{w}_{2}, \ldots, A \mathbf{w}_{n}\right) \\
= & \operatorname{tr}\left(d A A^{-1}\right) \operatorname{det}(A) \operatorname{det}\left(\mathbf{w}_{1}, \mathbf{w}_{2}, \ldots, \mathbf{w}_{n}\right) \\
= & \operatorname{det}(A) \operatorname{tr}\left(A^{-1} d A\right) \operatorname{det}\left(\mathbf{w}_{1}, \mathbf{w}_{2}, \ldots, \mathbf{w}_{n}\right)
\end{aligned}
$$

Hence,

$$
d(\operatorname{det} A)=\operatorname{det} A \operatorname{tr}\left(A^{-1} d A\right) .
$$

It is now sufficient to take $d=\tilde{\delta}$ and to apply this result to $\operatorname{det}(F)$. The Lemma has been proved.

In particular, the Lemma and (3) imply :

$$
\tilde{\delta} \rho=-\rho \operatorname{div}(\delta \mathbf{x}), \quad \hat{\delta} \rho=-\operatorname{div}(\rho \delta \mathbf{x}) .
$$

\section{Variation of entropy}


The entropy per unit mass $\eta$ is conserved along trajectories, so it is a function only of the Lagrangian coordinates : $\eta=\eta_{0}(\mathbf{X})$. Hence, its Lagrangian and Eulerian variations are :

$$
\tilde{\delta} \eta=0, \quad \hat{\delta} \eta=-\nabla \eta \cdot \delta \mathbf{x}
$$

\section{Variation of velocity}

The variation of the velocity is direct :

$$
\tilde{\delta} \mathbf{v}=\frac{\partial \delta \mathbf{x}}{\partial t}, \quad \hat{\delta} \mathbf{v}=\frac{D \delta \mathbf{x}}{D t}-\frac{\partial \mathbf{v}}{\partial \mathbf{x}} \delta \mathbf{x},
$$

where the material derivative $\frac{D}{D t}$ is defined as

$$
\frac{D}{D t}=\frac{\partial}{\partial t}+\mathbf{v} \cdot \nabla
$$

\section{$3 \quad$ Hamilton's principle}

Let us consider the Hamilton action :

$$
a=\int_{t_{0}}^{t_{1}} L d t,
$$

where $\left[t_{0}, t_{1}\right]$ is a time interval and $L$ is the corresponding Lagrangian. The Hamilton principle is : for any virtual motion satisfying

$$
\begin{gathered}
\boldsymbol{\Phi}_{t_{0}}(\mathbf{X}, \varepsilon)=\boldsymbol{\varphi}_{t_{0}}(\mathbf{X}), \quad \boldsymbol{\Phi}_{t_{1}}(\mathbf{X}, \varepsilon)=\boldsymbol{\varphi}_{t_{1}}(\mathbf{X}), \\
\boldsymbol{\Phi}_{t}(\mathbf{X}, \varepsilon)=\boldsymbol{\varphi}_{t}(\mathbf{X}), \quad \mathbf{X} \in \partial \mathcal{D}\left(t_{0}\right),
\end{gathered}
$$

and imposed constraints, the variation of the Hamilton action is stationary :

$$
\delta a=0
$$

Let us remark that (4), (5) imply

$$
\left.\delta \mathbf{x}\right|_{t=t_{0}}=\left.\delta \mathbf{x}\right|_{t=t_{1}}=0,\left.\quad \delta \mathbf{x}\right|_{\partial \mathcal{D}(t)}=0 .
$$

We will recall first the Lagrangians for classical models of continuum mechanics and the corresponding constraints.

\section{Lagrangian of an incompressible fluid}

$$
L=\int_{\mathcal{D}(t)} \frac{\rho|\mathbf{v}|^{2}}{2} d D
$$


The density $\rho$ and the velocity field $\mathbf{v}$ verify the constraints :

$$
\frac{D \rho}{D t}=0, \quad \operatorname{div}(\mathbf{v})=0 .
$$

\section{Lagrangian of a compressible fluid}

$$
L=\int_{\mathcal{D}(t)} \rho\left(\frac{|\mathbf{v}|^{2}}{2}-e(\rho, \eta)\right) d D
$$

The density $\rho$, the velocity field $\mathbf{v}$ and the entropy $\eta$ verify the constraints :

$$
\frac{D \rho}{D t}+\rho \operatorname{div}(\mathbf{v})=0, \quad \frac{D \eta}{D t}=0 .
$$

The specific internal energy $e(\rho, \eta)$ is a given function satisfying the Gibbs identity :

$$
\theta d \eta=d e+p d\left(\frac{1}{\rho}\right),
$$

where $\theta$ is the temperature and $p$ is the pressure.

Lagrangian of an elastic body

$$
L=\int_{\mathcal{D}(t)} \rho\left(\frac{|\mathbf{v}|^{2}}{2}-e(G, \eta)\right) d D .
$$

Here

$$
G=\left(F F^{T}\right)^{-1}=\left(F^{T}\right)^{-1} F^{-1}
$$

is the Finger tensor. In the case of isotropic solids the energy is a function of the invariants of $G$. This Lagrangian is suitable for the Eulerian description of solids. Another possibility commonly used in the literature is to consider the specific energy in the form $e(C, \eta)$ where $C=F^{T} F$ is the right CauchyGreen deformation tensor.

\section{Governing equations of classical continua}

\subsection{Incompressible fluids}

Let

$$
a=\int_{t_{0}}^{t_{1}} L d t=\int_{t_{0}}^{t_{1}} \int_{\mathcal{D}(t)} \frac{\rho|\mathbf{v}|^{2}}{2} d D
$$

The constraints

$$
\frac{D \rho}{D t}=0, \quad \operatorname{div}(\mathbf{v})=0
$$


can be written in the form

$$
\rho=\rho_{0}(\mathbf{X}), \quad \operatorname{det} F=1 .
$$

Hence

$$
\hat{\delta} \rho=-\nabla \rho \cdot \delta \mathbf{x}, \quad \operatorname{div}(\delta \mathbf{x})=0 .
$$

To satisfy the last constraint we take

$$
\delta \mathbf{x}=\operatorname{rot}(\mathbf{A})
$$

where $\mathbf{A}$ is a smooth vector field vanishing at the boundary $\mathcal{D}(t)$ :

$$
\left.\mathbf{A}\right|_{\partial \mathcal{D}(t)}=0 .
$$

The variation of the Hamilton action is

$$
\begin{gathered}
\delta a=\left.\frac{d}{d \varepsilon}\right|_{\varepsilon=0} \int_{t_{0}}^{t_{1}} \int_{\mathcal{D}(t)} \frac{\hat{\rho}|\hat{\mathbf{v}}|^{2}}{2} d D=\int_{t_{0}}^{t_{1}} \int_{\mathcal{D}(t)} \hat{\delta}\left(\frac{\rho|\mathbf{v}|^{2}}{2}\right) d D \\
=\int_{t_{0}}^{t_{1}} \int_{\mathcal{D}(t)}\left(\hat{\delta} \rho \frac{|\mathbf{v}|^{2}}{2}+\rho \mathbf{v} \cdot \hat{\delta} \mathbf{v}\right) d D \\
=\int_{t_{0}}^{t_{1}} \int_{\mathcal{D}(t)}\left(-\frac{|\mathbf{v}|^{2}}{2} \nabla \rho \cdot \delta \mathbf{x}+\rho \mathbf{v} \cdot\left(\frac{D \delta \mathbf{x}}{D t}-\frac{\partial \mathbf{v}}{\partial \mathbf{x}} \delta \mathbf{x}\right)\right) d D \\
=\int_{t_{0}}^{t_{1}} \int_{\mathcal{D}(t)}\left(-\nabla\left(\frac{\rho|\mathbf{v}|^{2}}{2}\right) \cdot \delta \mathbf{x}+\rho \mathbf{v} \cdot \frac{D \delta \mathbf{x}}{D t}\right) d D \\
=\int_{t_{0}}^{t_{1}} \int_{\mathcal{D}(t)}\left(-\nabla\left(\frac{\rho|\mathbf{v}|^{2}}{2}\right) \cdot \delta \mathbf{x}+\rho \mathbf{v} \cdot\left(\frac{\partial \delta \mathbf{x}}{\partial t}+\frac{\partial \delta \mathbf{x}}{\partial \mathbf{x}} \mathbf{v}\right)\right) d D \\
=\int_{t_{0}}^{t_{1}} \int_{\mathcal{D}(t)}\left(\frac{\partial(\rho \mathbf{v} \cdot \delta \mathbf{x})}{\partial t}+\operatorname{div}\left((\rho \mathbf{v} \otimes \mathbf{v}) \cdot \delta \mathbf{x}-\frac{\rho|\mathbf{v}|^{2}}{2} \delta \mathbf{x}\right)\right) d D \\
-\int_{t_{0}}^{t_{1}} \int_{\mathcal{D}(t)}\left(\frac{\partial \rho \mathbf{v}}{\partial t}+\operatorname{div}(\rho \mathbf{v} \otimes \mathbf{v})\right) \cdot \delta \mathbf{x} d D
\end{gathered}
$$

Using the Gauss-Ostrogradski formula and taking into account the fact that $\delta \mathbf{x}$ vanishes at $\partial \mathcal{D}(t)$ and $\left.\delta \mathbf{x}\right|_{t=t_{0}}=\left.\delta \mathbf{x}\right|_{t=t_{1}}=0$, we finally get

$$
\delta a=-\int_{t_{0}}^{t_{1}} \int_{\mathcal{D}(t)}\left(\frac{\partial \rho \mathbf{v}}{\partial t}+\operatorname{div}(\rho \mathbf{v} \otimes \mathbf{v})\right) \cdot \delta \mathbf{x} d D
$$


Let us remark that for any function $f$

$$
\begin{gathered}
\int_{t_{0}}^{t_{1}} \int_{\mathcal{D}(t)} \nabla f \cdot \operatorname{rot}(\mathbf{A}) d D=\int_{t_{0}}^{t_{1}} \int_{\mathcal{D}(t)} \operatorname{div}(f \operatorname{rot}(\mathbf{A})) d D \\
=\int_{t_{0}}^{t_{1}} \int_{\partial \mathcal{D}(t)} f \operatorname{rot}(\mathbf{A}) \cdot \mathbf{n} d D=0
\end{gathered}
$$

if

$$
\operatorname{rot}(\mathbf{A}) \cdot \mathbf{n}=\mathbf{0} .
$$

Here $\mathbf{n}$ is the external unit normal vector to $\partial \mathcal{D}(t)$. But the last condition is verified if the field $\mathbf{A}$ is orthogonal to $\partial \mathcal{D}(t)$ :

$$
\mathbf{A} \cdot \mathbf{s}=\mathbf{0}
$$

for any vector $\mathbf{s}$ tangent to $\partial \mathcal{D}(t)$. But

$$
\left.\mathbf{A}\right|_{\partial \mathcal{D}(t)}=0 .
$$

Hence

$$
\begin{gathered}
\delta a=-\int_{t_{0}}^{t_{1}} \int_{\mathcal{D}(t)}\left(\frac{\partial \rho \mathbf{v}}{\partial t}+\operatorname{div}(\rho \mathbf{v} \otimes \mathbf{v})\right) \cdot \delta \mathbf{x} d D \\
=-\int_{t_{0}}^{t_{1}} \int_{\mathcal{D}(t)}\left(\frac{\partial \rho \mathbf{v}}{\partial t}+\operatorname{div}(\rho \mathbf{v} \otimes \mathbf{v})\right) \cdot \operatorname{rot}(\mathbf{A}) d D .
\end{gathered}
$$

Hence, there exists a scalar function $p$ called pressure such that

$$
\frac{\partial \rho \mathbf{v}}{\partial t}+\operatorname{div}(\rho \mathbf{v} \otimes \mathbf{v})=-\nabla p
$$

So, we have obtained the Euler equations of incompressible non-homogeneous fluids :

$$
\begin{gathered}
\frac{\partial \rho \mathbf{v}}{\partial t}+\operatorname{div}(\rho \mathbf{v} \otimes \mathbf{v}+p \mathbf{I})=0, \\
\frac{D \rho}{D t}=0, \quad \operatorname{div}(\mathbf{v})=0 .
\end{gathered}
$$

When the mass forces $\mathbf{f}$ are added, the equations become

$$
\begin{gathered}
\frac{\partial \rho \mathbf{v}}{\partial t}+\operatorname{div}(\rho \mathbf{v} \otimes \mathbf{v}+p \mathbf{I})=\rho \mathbf{f}, \\
\frac{D \rho}{D t}=0, \quad \operatorname{div}(\mathbf{v})=0 .
\end{gathered}
$$

In the case of potential forces $(\mathbf{f}=-\nabla \boldsymbol{\Psi}(\mathbf{x}))$ the equations admit the energy conservation law :

$$
\frac{\partial}{\partial t}\left(\rho\left(\frac{|\mathbf{v}|^{2}}{2}+\boldsymbol{\Phi}\right)\right)+\operatorname{div}\left(\rho \mathbf{v}\left(\frac{|\mathbf{v}|^{2}}{2}+\mathbf{\Phi}\right)+p \mathbf{v}\right)=0 .
$$




\subsection{Compressible fluids}

Let us consider the Hamilton action for compressible fluids :

$$
a=\int_{t_{0}}^{t_{1}} L d t=\int_{t_{0}}^{t_{1}} \int_{\mathcal{D}(t)}\left(\frac{\rho|\mathbf{v}|^{2}}{2}-\rho e(\rho, \eta)\right) d D
$$

The constraints

$$
\frac{D \rho}{D t}+\rho \operatorname{div}(\mathbf{v})=0, \quad \frac{D \eta}{D t}=0,
$$

can by rewritten in the Lagrangian coordinates in the form

$$
\rho \operatorname{det} F=\rho_{0}(\mathbf{X}), \quad \eta=\eta_{0}(\mathbf{X}) .
$$

Hence

$$
\hat{\delta} \rho=-\operatorname{div}(\rho \delta \mathbf{x}), \quad \hat{\delta} \eta=-\nabla \eta \cdot \delta \mathbf{x} .
$$

The variation of the Hamilton action is :

$$
\begin{gathered}
\delta a=\left.\frac{d}{d \varepsilon}\right|_{\varepsilon=0} \int_{t_{0}}^{t_{1}} \int_{\mathcal{D}(t)}\left(\frac{\hat{\rho}|\hat{\mathbf{v}}|^{2}}{2}-\hat{\rho} e(\hat{\rho}, \hat{\eta})\right) d D \\
=\int_{t_{0}}^{t_{1}} \int_{\mathcal{D}(t)} \hat{\delta}\left(\frac{\rho|\mathbf{v}|^{2}}{2}-\rho e(\rho, \eta)\right) d D \\
=\int_{t_{0}}^{t_{1}} \int_{\mathcal{D}(t)}\left(\hat{\delta} \rho\left(\frac{|\mathbf{v}|^{2}}{2}-e-\frac{p}{\rho}\right)+\rho \mathbf{v} \cdot \hat{\delta} \mathbf{v}-\frac{\partial e(\rho, \eta)}{\partial \eta} \hat{\delta} \eta\right) d D \\
=-\int_{t_{0}}^{t_{1}} \int_{\mathcal{D}(t)} d i v(\rho \delta \mathbf{x})\left(\frac{|\mathbf{v}|^{2}}{2}-\left(e+\frac{p}{\rho}\right)\right) d D \\
\int_{t_{0}}^{t_{1}} \int_{\mathcal{D}(t)}\left(\rho \mathbf{v} \cdot\left(\frac{D \delta \mathbf{x}}{D t}-\frac{\partial \mathbf{v}}{\partial \mathbf{x}} \delta \mathbf{x}\right)+\frac{\partial e(\rho, \eta)}{\partial \eta} \nabla \eta \cdot \delta \mathbf{x}\right) d D \\
=\int_{t_{0}}^{t_{1}} \int_{\mathcal{D}(t)} \rho \delta \mathbf{x} \cdot \nabla\left(\frac{|\mathbf{v}|^{2}}{2}-\left(e+\frac{p}{\rho}\right)\right) d D \\
+\int_{t_{0}}^{t_{1}} \int_{\mathcal{D}(t)}\left(\rho \mathbf{v} \cdot\left(\frac{D \delta \mathbf{x}}{D t}-\frac{\partial \mathbf{v}}{\partial \mathbf{x}} \delta \mathbf{x}\right)+\frac{\partial e(\rho, \eta)}{\partial \eta} \nabla \eta \cdot \delta \mathbf{x}\right) d D \\
=-\int_{t_{0}}^{t_{1}} \int_{\mathcal{D}(t)} \rho \delta \mathbf{x} \cdot \nabla\left(e+\frac{p}{\rho}\right) d D
\end{gathered}
$$




$$
\begin{gathered}
+\int_{t_{0}}^{t_{1}} \int_{\mathcal{D}(t)}\left(\rho \mathbf{v} \cdot\left(\frac{\partial \delta \mathbf{x}}{\partial t}+\frac{\partial \delta \mathbf{x}}{\partial \mathbf{x}} \mathbf{v}\right)+\frac{\partial e(\rho, \eta)}{\partial \eta} \nabla \eta \cdot \delta \mathbf{x}\right) d D \\
=\int_{t_{0}}^{t_{1}} \int_{\mathcal{D}(t)}\left(-\rho \delta \mathbf{x} \cdot \frac{\nabla p}{\rho}+\rho \mathbf{v} \cdot\left(\frac{\partial \delta \mathbf{x}}{\partial t}+\frac{\partial \delta \mathbf{x}}{\partial \mathbf{x}} \mathbf{v}\right)\right) d D \\
=\int_{t_{0}}^{t_{1}} \int_{\mathcal{D}(t)}-\left(\frac{\partial \rho \mathbf{v}}{\partial t}+d i v(\rho \mathbf{v} \otimes \mathbf{v})+\nabla p\right) \cdot \delta \mathbf{x} d D=0 .
\end{gathered}
$$

In this derivation, we have used the Gauss-Ostrogradski formula and have taken into account the fact that $\delta \mathbf{x}$ vanishes at $\partial \mathcal{D}(t)$ and $\left.\delta \mathbf{x}\right|_{t=t_{0}}=$ $\left.\delta \mathbf{x}\right|_{t=t_{1}}=0$. So, we have obtained the Euler equations of compressible fluids

$$
\begin{gathered}
\frac{\partial \rho \mathbf{v}}{\partial t}+\operatorname{div}(\rho \mathbf{v} \otimes \mathbf{v}+p \mathbf{I})=0 \\
\frac{D \rho}{D t}+\rho \operatorname{div}(\mathbf{v})=0 \\
\frac{D \eta}{D t}=0
\end{gathered}
$$

These equations admit the energy conservation law :

$$
\frac{\partial}{\partial t}\left(\rho\left(\frac{|\mathbf{v}|^{2}}{2}+e(\rho, \eta)\right)\right)+\operatorname{div}\left(\rho \mathbf{v}\left(\frac{|\mathbf{v}|^{2}}{2}+e(\rho, \eta)\right)+p \mathbf{v}\right)=0
$$

\subsection{Isotropic elastic bodies}

Let

$$
L=\int_{\mathcal{D}(t)} \rho\left(\frac{|\mathbf{v}|^{2}}{2}-e(G, \eta)\right) d D
$$

Here

$$
G=\left(F F^{T}\right)^{-1}=F^{-T} F^{-1}
$$

is the Finger tensor. The tensor $G$ is more convenient for the Eulerian formulation of governing equations of isotropic elastic materials. Let us recall that the material is isotropic if for any orthogonal transformation $O$

$$
e\left(O G O^{T}, \eta\right)=e(G, \eta)
$$

Hence, the internal energy depends only on the invariants of $G$. In particular, we choose such invariants in the form :

$$
J_{i}=\operatorname{tr}\left(G^{i}\right), i=1,2,3 .
$$


Let $\mathbf{X}=\left(X^{\alpha}\right)$ be the Lagrangian coordinates, $\alpha=1,2,3, \mathbf{x}=\left(x^{i}\right)$ be the Eulerian coordinates, $i=1,2,3$. We introduce the curvilinear cobasis

$$
\mathbf{e}^{\alpha}=\nabla X^{\alpha}, \quad \alpha=1,2,3
$$

which is dual to the natural curvilinear basis

$$
\mathbf{e}_{\alpha}=\frac{\partial \mathbf{x}}{\partial X^{\alpha}}, \quad \alpha=1,2,3
$$

In particular, the vector $\mathbf{e}^{\alpha}$ is the $\alpha$ - th column of $F^{-T}$ :

$$
\begin{aligned}
& F^{-T}=\left(\begin{array}{ccc}
\frac{\partial X^{1}}{\partial x^{1}} & \frac{\partial X^{2}}{\partial x^{1}} & \frac{\partial X^{3}}{\partial x^{1}} \\
\frac{\partial X^{1}}{\partial x^{2}} & \frac{\partial X^{2}}{\partial x^{2}} & \frac{\partial X^{3}}{\partial x^{2}} \\
\frac{\partial X^{1}}{\partial x^{3}} & \frac{\partial X^{2}}{\partial x^{3}} & \frac{\partial X^{3}}{\partial x^{3}}
\end{array}\right)=\left(\begin{array}{lll}
\mathbf{e}^{1}, & \mathbf{e}^{2}, & \mathbf{e}^{3}
\end{array}\right) \\
& =\left(\begin{array}{lll}
e_{1}^{1} & e_{1}^{2} & e_{1}^{3} \\
e_{2}^{1} & e_{2}^{2} & e_{2}^{3} \\
e_{3}^{1} & e_{3}^{2} & e_{3}^{3}
\end{array}\right), \quad e_{j}^{\alpha}=\left(\mathbf{e}^{\alpha}\right)_{j}=\frac{\partial X^{\alpha}}{\partial x^{j}} .
\end{aligned}
$$

The scalar product of these vectors satisfies

$$
\mathbf{e}^{\beta} \cdot \mathbf{e}_{\alpha}=\delta_{\alpha}^{\beta}
$$

where $\delta_{\alpha}^{\beta}$ are the Kronecker symbols. With these definitions

$$
G=\sum_{\alpha=1}^{3} \mathbf{e}^{\alpha} \otimes \mathbf{e}^{\alpha}, \quad G^{-1}=\sum_{\alpha=1}^{3} \mathbf{e}_{\alpha} \otimes \mathbf{e}_{\alpha} .
$$

Since

$$
\frac{D X^{\alpha}}{D t}=0
$$

we have by taking the gradient of that equation :

$$
\frac{\partial \mathbf{e}^{\alpha}}{\partial t}+\nabla\left(\mathbf{v} \cdot \mathbf{e}^{\alpha}\right)=0, \quad \operatorname{rot}^{\alpha}=0
$$

In particular, equations (7) and (8) imply that

$$
\frac{D G}{D t}+G \frac{\partial \mathbf{v}}{\partial \mathbf{x}}+\left(\frac{\partial \mathbf{v}}{\partial \mathbf{x}}\right)^{T} G=0 .
$$

Remark 1. It is interesting to note that the left hand side of this equation can be considered as an objective derivative. Indeed, let $O(t)$ be a 
time dependent orthogonal transformation : $O(t) O(t)^{T}=I$. Consider new variables denoted with primes :

$$
\mathbf{x}^{\prime}=O(t) \mathbf{x}, \mathbf{v}^{\prime}=\frac{d O(t)}{d t} \mathbf{x}+O(t) \mathbf{v} .
$$

The aim is to show that the equation for

$$
G^{\prime}=O(t) G O^{T}(t)
$$

will be the same as for $G$. We get :

$$
\begin{gathered}
\frac{D G}{D t}=\frac{\partial G}{\partial t}+\frac{\partial G}{\partial \mathbf{x}} \mathbf{v} \\
=\frac{\partial\left(O^{T}(t) G^{\prime} O(t)\right)}{\partial t^{\prime}}+\frac{\partial\left(O^{T}(t) G^{\prime} O(t)\right)}{\partial \mathbf{x}^{\prime}} \frac{\partial \mathbf{x}^{\prime}}{\partial t} \\
+\frac{\partial\left(O^{T}(t) G^{\prime} O(t)\right)}{\partial \mathbf{x}^{\prime}} \frac{\partial \mathbf{x}^{\prime}}{\partial \mathbf{x}}\left(O^{T}(t) \mathbf{v}^{\prime}-O^{T}(t) \frac{d O(t)}{d t} O^{T}(t) \mathbf{x}^{\prime}\right) \\
=\frac{\partial\left(O^{T}(t) G^{\prime} O(t)\right)}{\partial t^{\prime}}+\frac{\partial\left(O^{T}(t) G^{\prime} O(t)\right)}{\partial \mathbf{x}^{\prime}} \frac{d O(t)}{d t} O^{T}(t) \mathbf{x}^{\prime} \\
+\frac{\partial\left(O^{T}(t) G^{\prime} O(t)\right)}{\partial \mathbf{x}^{\prime}}\left(\mathbf{v}^{\prime}-\frac{d O(t)}{d t} O^{T}(t) \mathbf{x}^{\prime}\right) \\
=\frac{\partial\left(O^{T}(t) G^{\prime} O(t)\right)}{\partial t^{\prime}}+\frac{\partial\left(O^{T}(t) G^{\prime} O(t)\right)}{\partial \mathbf{x}^{\prime}} \mathbf{v}^{\prime} \\
=\frac{d O^{T}(t)}{d t} G^{\prime} O(t)+O^{T}(t) G^{\prime} \frac{d O(t)}{d t}+O^{T}(t) \frac{D^{\prime} G^{\prime}}{D t^{\prime}} O(t) .
\end{gathered}
$$

Hence

$$
\begin{gathered}
\frac{D G}{D t}+G \frac{\partial \mathbf{v}}{\partial \mathbf{x}}+\left(\frac{\partial \mathbf{v}}{\partial \mathbf{x}}\right)^{T} G= \\
=\frac{d O^{T}(t)}{d t} G^{\prime} O(t)+O^{T}(t) G^{\prime} \frac{d O(t)}{d t}+O^{T}(t) \frac{D^{\prime} G^{\prime}}{D t^{\prime}} O(t) \\
+O^{T}(t) G^{\prime} O(t)\left(O^{T}(t) \frac{\partial \mathbf{v}^{\prime}}{\partial \mathbf{x}^{\prime}} O(t)-O^{T}(t) \frac{d O(t)}{d t}\right) \\
+\left(O^{T}(t) \frac{\partial \mathbf{v}^{\prime}}{\partial \mathbf{x}^{\prime}} O(t)-O^{T}(t) \frac{d O(t)}{d t}\right) O^{T}(t) G^{\prime} O(t) \\
=O^{T}(t)\left(\frac{D^{\prime} G^{\prime}}{D t^{\prime}}+G^{\prime} \frac{\partial \mathbf{v}^{\prime}}{\partial \mathbf{x}^{\prime}}+\left(\frac{\partial \mathbf{v}^{\prime}}{\partial \mathbf{x}^{\prime}}\right)^{T} G^{\prime}\right) O(t) .
\end{gathered}
$$


Finally,

$$
\begin{gathered}
\frac{D^{\prime} G^{\prime}}{D t^{\prime}}+G^{\prime} \frac{\partial \mathbf{v}^{\prime}}{\partial \mathbf{x}^{\prime}}+\left(\frac{\partial \mathbf{v}^{\prime}}{\partial \mathbf{x}^{\prime}}\right)^{T} G^{\prime} \\
=O(t)\left(\frac{D G}{D t}+G \frac{\partial \mathbf{v}}{\partial \mathbf{x}}+\left(\frac{\partial \mathbf{v}}{\partial \mathbf{x}}\right)^{T} G\right) O^{T}(t) .
\end{gathered}
$$

Remark 2. The differential constraint $\operatorname{rote}^{\alpha}=0$ in (8) is compatible with the evolution equation for $\mathbf{e}^{\alpha}$ : if $\operatorname{rot}^{\alpha}$ vanishes at time $t=0$, it vanishes at all $t>0$. This constraint is automatically satisfied in the onedimensional case. In the multi-dimensional case equation (8) can also be replaced by

$$
\frac{\partial \mathbf{e}^{\alpha}}{\partial t}+\nabla\left(\mathbf{v} \cdot \mathbf{e}^{\alpha}\right)=-\operatorname{rote}^{\alpha} \wedge \mathbf{v} .
$$

We will obtain now the expression for the stress tensor. Let $e$ be the specific internal energy :

$$
e=e(G, \eta)
$$

where $\eta$ is the specific entropy. Let us consider the variation of the internal energy $\mathcal{E}_{i}$ at fixed value of $\eta$ :

$$
\delta \mathcal{E}_{i}=\delta \int_{\mathcal{D}(t)} \rho e(G, \eta) d D
$$

We have

$$
\begin{gathered}
\delta \mathcal{E}_{i}=\delta \int_{\mathcal{D}(t)} \rho e(G, \eta) d D=\int_{\mathcal{D}(0)} \rho_{0} \tilde{\delta} e(G, \eta) d D_{0} \\
=\int_{\mathcal{D}(0)} \rho_{0} \operatorname{tr}\left(\frac{\partial e}{\partial G} \tilde{\delta} G\right) d D_{0}
\end{gathered}
$$

The matrix $\partial e / \partial G$ is symmetric. Since

$$
\begin{gathered}
\tilde{\delta} G=\left(\tilde{\delta} F^{-1}\right)^{T} F^{-1}+\left(F^{-1}\right)^{T} \tilde{\delta} F^{-1} \\
=-\left(F^{-1} \tilde{\delta} F F^{-1}\right)^{T} F^{-1}-\left(F^{-1}\right)^{T} F^{-1} \tilde{\delta} F F^{-1} \\
=-\left(\frac{\partial \delta \mathbf{x}}{\partial \mathbf{x}}\right)^{T} G-G \frac{\partial \delta \mathbf{x}}{\partial \mathbf{x}}
\end{gathered}
$$

we obtain

$$
\delta \mathcal{E}_{i}=-\int_{\mathcal{D}(0)} 2 \rho_{0} \operatorname{tr}\left(\frac{\partial e}{\partial G} G \frac{\partial \delta \mathbf{x}}{\partial \mathbf{x}}\right) d D_{0}
$$




$$
=\int_{\mathcal{D}(t)} \operatorname{tr}\left(\sigma \frac{\partial \delta \mathbf{x}}{\partial \mathbf{x}}\right) d D
$$

The tensor

$$
\sigma=-2 \rho \frac{\partial e}{\partial G} G
$$

is called the Murnaghan stress tensor (Godunov, 1978, Godunov and Romenskii, 2003). It is symmetric, if $e$ is isotropic. Indeed, in the isotropic case, $e$ depends only on the invariants of $G$ :

$$
J_{i}=\operatorname{tr}\left(G^{i}\right), \quad i=1,2,3
$$

In particular, the determinant of $G$ (denoted by $|G|$ ) can be expressed in terms of $J_{i}$ in the form

$$
|G|=\frac{2 J_{3}-3 J_{1} J_{2}+J_{1}^{3}}{6}
$$

It can be proved that

$$
\frac{\partial J_{i}}{\partial G}=i G^{i-1}, i=1,2,3
$$

In particular (see also the Lemma)

$$
\frac{\partial|G|}{\partial G}=|G| G^{-1}
$$

Hence

$$
\sigma=-2 \rho \frac{\partial e}{\partial G} G=-2 \rho\left(\frac{\partial e}{\partial J_{1}} I+2 \frac{\partial e}{\partial J_{2}} G+3 \frac{\partial e}{\partial J_{3}} G^{2}\right) G=-2 \rho G \frac{\partial e}{\partial G}=\sigma^{T}
$$

Here the density $\rho$ is expressed by

$$
\rho=\rho_{0}|G|^{1 / 2}
$$

EOS formulation in separable form It is natural to present the energy in a separable form : the energy is the sum of a "hydrodynamic" part and an "elastic" part :

$$
e=e^{h}(\rho, \eta)+e^{e}(g)
$$

where

$$
g=\frac{G}{|G|^{1 / 3}} .
$$


The hydrodynamic part of the energy $e^{h}(\rho, \eta)$ can be taken in the form of stiffened gas equation of state :

$$
e^{h}=\frac{p+\gamma p_{\infty}}{\rho(\gamma-1)}, \quad p+p_{\infty}=A \exp \left(\frac{\eta-\eta_{0}}{c_{v}}\right) \rho^{\gamma}, \quad \eta_{0}=\text { const }, \quad A=\text { const }
$$

Here $\gamma$ is the polytropic exponent, $p_{\infty}$ is a constant, $c_{v}$ is the specific heat at constant volume.

The elastic part of the internal energy $e^{e}$ depends only on $g$. The tensor $g$ has a unit determinant, so it is unaffected by the volume change. This idea to take the arguments of the internal energy in this form was first proposed by Gouin and Debieve (1986) (see also Plohr and Plohr, 2005), but for the dependence of the energy on the right Cauchy-Green tensor. The simplest example of the elastic energy is

$$
e^{e}(g, \eta)=\frac{\mu_{s}}{4 \rho_{0}} \operatorname{tr}\left((g-I)^{2}\right)=\frac{\mu_{s}}{4 \rho_{0}}\left(\frac{J_{2}}{|G|^{2 / 3}}-\frac{2 J_{1}}{|G|^{1 / 3}}+3\right)
$$

where $\mu_{s}$ is the shear modulus. The stress tensor will be then

$$
\sigma=-2 \rho \frac{\partial e}{\partial G} G=-p I+S, \quad \operatorname{tr}(S)=0
$$

where the deviatoric part is

$$
S=-\mu_{s} \frac{\rho}{\rho_{0}}\left(\frac{1}{|G|^{2 / 3}}\left(G^{2}-\frac{J_{2}}{3} I\right)-\frac{1}{|G|^{1 / 3}}\left(G-\frac{J_{1}}{3} I\right)\right)
$$

and the thermodynamic pressure is

$$
p=\rho^{2} \frac{\partial e^{h}}{\partial \rho}
$$

The elastic part of the energy has no influence on the pressure, it is determined only by the hydrodynamic part. In the case of small displacements, we obtain the classical Hooke law. 
Governing equations The governing equations can be written in the form :

$$
\begin{aligned}
& \frac{\partial \mathbf{e}^{\alpha}}{\partial t}+\nabla\left(\mathbf{v} \cdot \mathbf{e}^{\alpha}\right)=0, \quad \operatorname{rot}^{\alpha}=0, \\
& \frac{\partial \rho}{\partial t}+\operatorname{div}(\rho \mathbf{v})=0, \\
& \frac{\partial \rho \mathbf{v}}{\partial t}+\operatorname{div}(\rho \mathbf{v} \otimes \mathbf{v}-\sigma)=0, \\
& \frac{\partial\left(\rho\left(e+\frac{\mathbf{v} \cdot \mathbf{v}}{2}\right)\right)}{\partial t}+\operatorname{div}\left(\rho \mathbf{v}\left(e+\frac{\mathbf{v} \cdot \mathbf{v}}{2}\right)-\sigma \mathbf{v}\right)=0 .
\end{aligned}
$$

For the energy in the form (10), 11), the equations are hyperbolic at least in the one-dimensional case. The proof will be given in a more general situation involving solid-fluid mixtures.

\section{Multiphase flow modeling: general definitions}

Consider a multiphase mixture of two compressible components. Each $a$ th component $(a=1,2)$ has its own phase average characteristics: the velocity $\mathbf{v}_{a}$; the density $\rho_{a}$; entropy $\eta_{a}$, the internal energy per unit mass $e_{a}\left(\rho_{a}, \eta_{a}\right)$, the temperature $\theta_{a}$ etc. The apparent densities $\bar{\rho}_{a}$ are defined by $: \bar{\rho}_{a}=\alpha_{a} \rho_{a}$. The mixture density is : $\rho=\alpha_{1} \rho_{1}+\alpha_{2} \rho_{2}$. Here $\alpha_{a}$ is the volume fraction of the $a$-th component, $\alpha_{1}+\alpha_{2}=1$.

The definition of the volume fraction is as follows. At each point $(t, \mathbf{x})$ consider a representative mixture volume $\Omega: \Omega=\Omega_{1} \cup \Omega_{2}$ where $\Omega_{a}$ is the volume occupied by the $\alpha-t h$ phase. Consider the characteristic function of the $a-t h$ phase :

$$
\chi_{a}\left(t, \mathbf{x}^{\prime}\right)=\left\{\begin{array}{c}
1, \text { if }\left(t, \mathbf{x}^{\prime}\right) \in \Omega_{a} \\
0, \text { if }\left(t, \mathbf{x}^{\prime}\right) \notin \Omega_{a}
\end{array}\right.
$$

Then

$$
\alpha_{a}(t, \mathbf{x})=\left\langle\chi_{a}\right\rangle=\frac{\int_{\Omega} \chi_{a}\left(t, \mathbf{x}^{\prime}\right) d \omega^{\prime}}{\operatorname{Vol}(\Omega)}=\frac{\operatorname{Vol}\left(\Omega_{a}\right)}{\operatorname{Vol}(\Omega)}
$$

The phase average characteristics are defined in standard way :

$$
\rho_{a}(t, \mathbf{x})=\frac{\int_{\Omega_{a}} \rho_{a}^{\prime}\left(t, \mathbf{x}^{\prime}\right) d \omega^{\prime}}{\operatorname{Vol}\left(\Omega_{a}\right)}
$$




$$
\mathbf{v}_{a}(t, \mathbf{x})=\frac{\int_{\Omega_{a}} \rho_{a}^{\prime}\left(t, \mathbf{x}^{\prime}\right) \mathbf{v}_{a}^{\prime}\left(t, \mathbf{x}^{\prime}\right) d \omega^{\prime}}{\int_{\Omega_{a}} \rho_{a}^{\prime}\left(t, \mathbf{x}^{\prime}\right) d \omega^{\prime}}
$$

etc. Other types of average can be introduced: time average, space-time average etc. (see, for example, Drew and Passman (1998), Ishii and Hibiki (2006)). The usual technique in obtaining the governing equations of multiphase flows is to multiply the system of balance laws by the characteristic function and average it. In particular, the mass conservation laws become :

$$
\frac{\partial\left(\alpha_{a} \rho_{a}\right)}{\partial t}+\operatorname{div}\left(\alpha_{a} \rho_{a} \mathbf{v}_{a}\right)=0 .
$$

However, applying the same averaging procedure to the conservation laws of momentum and energy, we immediately arrive to the closure problem : the balance equations contain more unknowns than equations. A possible remedy to that is to formulate the Lagrangian of the system directly in terms of average variables. And then to proceed in usual way by writing the corresponding Euler-Lagrange equations. The principal difficulty in applying such an approach is : how to determine this Lagrangian? At least two approaches can be used. The first is a phenomenological formulation of such a Lagrangian. The validation of such an approach should be established by comparing to experiments. The second possibility is an asymptotic modeling where the development with respect to a small parameter allows us to obtain an approximate Lagrangian from the exact one.

Also, it is not sufficient just to obtain the Euler-Lagrange equations : dissipation terms consistent with the second law of thermodynamics should be introduced to describe real physics.

\section{Equilibrium one-velocity model}

Quite often one can suppose that the average velocities of phases are equal $: \mathbf{v}_{1}=\mathbf{v}_{2}=\mathbf{v}$. We introduce the volume kinetic energy of the system :

$$
T=\sum_{a=1}^{2} \alpha_{a} \rho_{a} \frac{\left|\mathbf{v}_{a}\right|^{2}}{2}=\left(\sum_{a=1}^{2} \alpha_{a} \rho_{a}\right) \frac{|\mathbf{v}|^{2}}{2}=\rho \frac{|\mathbf{v}|^{2}}{2},
$$

and the potential energy

$$
W=\sum_{a=1}^{2} \alpha_{a} \rho_{a} e_{a}=\rho \sum_{a=1}^{2} Y_{a} e_{a}, \quad Y_{a}=\frac{\alpha_{a} \rho_{a}}{\rho}, \quad Y_{1}+Y_{2}=1 .
$$


The variable $Y_{a}$ is called mass fraction. The energies $e_{a}\left(\rho_{a}, \eta_{a}\right)$ verify the Gibbs identity:

$$
\theta_{a} d \eta_{a}=d e_{a}+p_{a} d\left(\frac{1}{\rho_{a}}\right)
$$

where $p_{a}$ are the pressures. The Lagrangian of the mixture is :

$$
L=\int_{\mathcal{D}(t)} \rho\left(\frac{|\mathbf{v}|^{2}}{2}-e\right) d D
$$

where we assume the additivity of the mixture energy defined as :

$$
e=Y_{1} e_{1}\left(\frac{Y_{1} \rho}{\alpha_{1}}, \eta_{1}\right)+Y_{2} e_{2}\left(\frac{Y_{2} \rho}{\alpha_{2}}, \eta_{2}\right) .
$$

The variation of the Hamilton action can be found under the usual constraints :

$$
\begin{gathered}
\frac{\partial \rho}{\partial t}+\operatorname{div}(\rho \mathbf{v})=0, \\
\frac{D Y_{1}}{D t}=0, \\
\frac{D \eta_{a}}{D t}=0, \quad a=1,2 .
\end{gathered}
$$

The novelty is that a new independent variable appears, the volume fraction, which is not related to the virtual displacements. Hence, an additional variation should be taken. The variations of the density, mass fraction and entropies are given by :

$$
\hat{\delta} \rho=-\operatorname{div}(\rho \delta \mathbf{x}), \quad \hat{\delta} \eta_{a}=-\nabla \eta_{a} \cdot \delta \mathbf{x}, \quad \hat{\delta} Y_{a}=-\nabla Y_{a} \cdot \delta \mathbf{x} .
$$

The corresponding variation of the Lagrangian can be found in the same way as for classical compressible fluids :

$$
\delta a=\int_{t_{0}}^{t_{1}} \int_{\mathcal{D}(t)}-\left(\frac{\partial \rho \mathbf{v}}{\partial t}+\operatorname{div}(\rho \mathbf{v} \otimes \mathbf{v})+\nabla p\right) \cdot \delta \mathbf{x} d D=0
$$

where

$$
p=\alpha_{1} p_{1}+\alpha_{2} p_{2}
$$

is the mixture pressure. It gives the momentum equation :

$$
\frac{\partial \rho \mathbf{v}}{\partial t}+\operatorname{div}(\rho \mathbf{v} \otimes \mathbf{v})+\nabla p=0
$$


Doing so, we have supposed that the volume fraction is fixed. Now, it is necessary to find the variation of the Lagrangian with respect to $\alpha_{1}$ :

$$
\begin{gathered}
\delta L=\int_{\mathcal{D}(t)} \rho\left(-Y_{1} \frac{\partial e_{1}}{\partial \rho_{1}}\left(-\frac{Y_{1} \rho}{\alpha_{1}^{2}}\right)-Y_{2} \frac{\partial e_{2}}{\partial \rho_{2}}\left(\frac{Y_{2} \rho}{\alpha_{2}^{2}}\right)\right) \delta \alpha_{1} d D \\
=\int_{\mathcal{D}(t)}\left(p_{1}-p_{2}\right) \delta \alpha_{1} d D=0 .
\end{gathered}
$$

Since it is valid for any $\delta \alpha_{1}$, it follows from here that

$$
p_{1}-p_{2}=0 \text {. }
$$

The equilibrium of velocities implies also the pressure equilibrium : $p=$ $p_{1}=p_{2}$. This is an algebraic equation for the volume fraction. The energy equation is a consequence of the mass conservation laws, the momentum equation and the entropy equations :

$$
\left(\rho\left(e+\frac{|\mathbf{v}|^{2}}{2}\right)\right)_{t}+\operatorname{div}\left(\rho \mathbf{v}\left(e+\frac{|\mathbf{v}|^{2}}{2}\right)+p \mathbf{v}\right)=0 .
$$

\subsection{What is the sound speed in multiphase flow models?}

The sound speeds of pure phases are determined as :

$$
c_{a}^{2}=\left.\frac{\partial p_{a}\left(\rho_{a}, \eta_{a}\right)}{\partial \rho_{a}}\right|_{\eta_{a}=\text { const }}, \quad a=1,2 .
$$

They correspond to the phase velocity of propagation of linear perturbations of the corresponding Euler equations linearized at rest. The corresponding sound speed $c_{w}$ in a two-fluid mixture is called Wood sound speed and is defined as

$$
\frac{1}{\rho c_{w}^{2}}=\frac{\alpha_{1}}{\rho_{1} c_{1}^{2}}+\frac{\alpha_{2}}{\rho_{2} c_{2}^{2}} .
$$

Consider first an euristic method of derivation of this formula. The definition of the specific mixture volume is :

$$
\tau=Y_{1} \tau_{1}+Y_{2} \tau_{2}
$$

where

$$
\tau=\frac{1}{\rho}, \quad \tau_{a}=\frac{1}{\rho_{a}}, \quad a=1,2 .
$$


Let us differentiate it with respect to the mixture pressure $p=p_{1}=p_{2}$ :

$$
\frac{d \tau}{d p}=-\frac{1}{\rho^{2} c_{w}^{2}}=Y_{1} \frac{d \tau_{1}}{d p_{1}}+Y_{2} \frac{d \tau_{2}}{d p_{2}}=-\frac{Y_{1}}{\rho_{1}^{2} c_{1}^{2}}-\frac{Y_{2}}{\rho_{2}^{2} c_{2}^{2}} .
$$

It implies immediately (14). Another way, more rigorous one, is to calculate the characteristic speeds of the governing system. For simplicity, we consider only one-dimensional case $(\mathbf{v}=(u), \mathbf{x}=(x))$. The equations are :

$$
\begin{gathered}
\frac{D \tau}{D t}-u_{x}=0, \\
\frac{D u}{D t}+\frac{p_{x}}{\rho}=0, \\
\frac{D Y_{1}}{D t}=0, \\
\frac{D \eta_{1}}{D t}=0, \\
\frac{D \eta_{2}}{D t}=0 .
\end{gathered}
$$

The mean specific volume is given by :

$$
\tau=\tau\left(p, \eta_{1}, \eta_{2}, Y_{1}\right)=Y_{1} \tau_{1}\left(p, \eta_{1}\right)+Y_{2} \tau_{2}\left(p, \eta_{2}\right)
$$

Obviously,

$$
\begin{gathered}
\frac{D \tau}{D t}=Y_{1} \frac{D \tau_{1}\left(p, \eta_{1}\right)}{D t}+Y_{2} \frac{D \tau_{1}\left(p, \eta_{1}\right)}{D t}= \\
-\left(\frac{Y_{1}}{\rho_{1}^{2} c_{1}^{2}}+\frac{Y_{2}}{\rho_{2}^{2} c_{2}^{2}}\right) \frac{D p}{D t}=-\left(\frac{\alpha_{1} \tau}{\rho_{1} c_{1}^{2}}+\frac{\alpha_{2} \tau}{\rho_{2} c_{2}^{2}}\right) \frac{D p}{D t} .
\end{gathered}
$$

Hence, the system is

$$
\begin{gathered}
\frac{D p}{D t}+\rho c_{w}^{2} u_{x}=0, \\
\frac{D u}{D t}+\frac{p_{x}}{\rho}=0 \\
\frac{D Y_{1}}{D t}=0 \\
\frac{D \eta_{1}}{D t}=0 \\
\frac{D \eta_{2}}{D t}=0
\end{gathered}
$$


System (15) can be rewritten in terms of unknowns $\mathbf{U}=\left(p, u, Y_{1}, \eta_{1}, \eta_{2}\right)^{T}$ :

$$
\mathbf{U}_{t}+A(\mathbf{U}) \mathbf{U}_{x}=0,
$$

where

$$
A(\mathbf{U})=\left(\begin{array}{ccccc}
u & \rho c_{w}^{2} & 0 & 0 & 0 \\
\frac{1}{\rho} & u & 0 & 0 & 0 \\
0 & 0 & u & 0 & 0 \\
0 & 0 & 0 & u & 0 \\
0 & 0 & 0 & 0 & u
\end{array}\right)
$$

The characteristic values of the matrix $A$ are

$$
\lambda_{1,2}=u \pm c_{w}, \quad \lambda_{3,4,5}=u .
$$

On can see that the system is hyperbolic (obviously, the eigenvalue $\lambda=u$ of multiplicity three has exactly three eigenvectors).

The Wood sound speed $c_{w}$ has very interesting physical properties. Indeed, let us fix the phase densities and entropies and plot the Wood sound speed as a function of the volume fraction.

$$
c_{w}=\frac{1}{\sqrt{\left(\alpha_{1} \rho_{1}+\alpha_{2} \rho_{2}\right)\left(\frac{\alpha_{1}}{\rho_{1} c_{1}^{2}}+\frac{\alpha_{2}}{\rho_{2} c_{2}^{2}}\right)}} .
$$

Its minimal value is much lower than the sound speeds in either medium. For example, for the "air-water" mixture with $c_{1}=330 \mathrm{~m} / \mathrm{s}, \rho_{1}=1,24$ $\mathrm{kg} / \mathrm{m}^{3}, c_{2}=1500 \mathrm{~m} / \mathrm{s}, \rho_{2}=1000 \mathrm{~kg} / \mathrm{m}^{3}$ the minimal sound speed is about $23 \mathrm{~m} / \mathrm{s}$. This very surprising fact has also been validated experimentally (Micaelli, 1982).

The model (15) we have derived is usually called "5 equations model" (see, for example, Kapila et al. (2001) for the derivation of this model from a more general two-velocity model). As we have seen, it is hyperbolic. However, it is not in conservative form. The last renders questionable a formal determination of the Rankine-Hugoniot relations for this model. A possible solution to this problem can be found in Saurel et al. (2007).

\section{Nonequilibrium one-velocity model}

The introduction of dissipation is always a phenomenological procedure which however should satisfy the entropy inequality. A natural relaxation equation can be written to replace the algebraic relation $p_{1}-p_{2}=0$. Introducing the dissipation function

$$
\mathcal{D}=\frac{1}{2 \mu}\left(\frac{D \alpha_{1}}{D t}\right)^{2}
$$




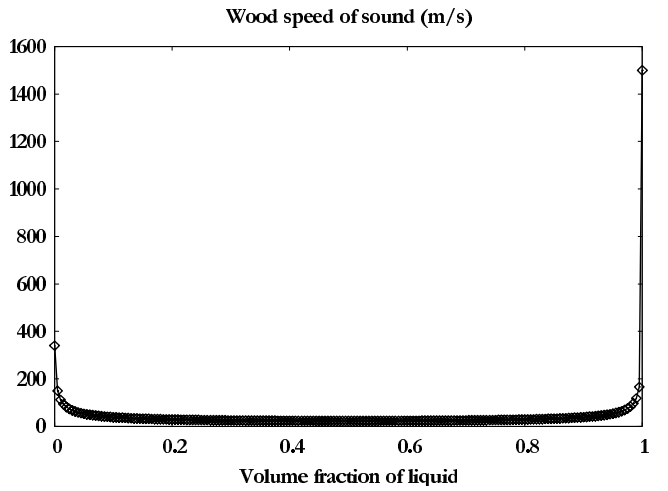

Figure 1. The minimal value of the Wood sound speed is much lower than the sound speeds in either components.

one can replace the equilibrium condition by the following one :

$$
p_{1}-p_{2}=\frac{\partial \mathcal{D}}{\partial\left(\frac{D \alpha_{1}}{D t}\right)}=\frac{1}{\mu} \frac{D \alpha_{1}}{D t} .
$$

Here $\mu$ is some constant. The physical sense of this equation is clear : the volume fraction changes due to the pressure difference. This equation is the simplest one. In more general case it may also contain inertia terms (see Gavrilyuk and Saurel (2002) and Saurel, Gavrilyuk and Renaud (2003) for details). The equations for entropies should satisfy the entropy inequality in the form :

$$
\frac{\partial(\rho \eta)}{\partial t}+\operatorname{div}(\rho \eta \mathbf{v}) \geq 0
$$

where $\eta$ is the mixture entropy defined by

$$
\rho \eta=\alpha_{1} \rho_{1} \eta_{1}+\alpha_{2} \rho_{2} \eta_{2} .
$$

We take the equations for the entropies in the form :

$$
\alpha_{a} \rho_{a} \theta_{a} \frac{D \eta_{a}}{D t}=f_{a}, \quad a=1,2
$$

where $f_{a}$ should be chosen to satisfy the entropy inequality (16). The production terms $f_{a}$ should also be compatible with the energy equation (13). Developing the energy equation one can obtain

$$
\frac{D e}{D t}+p \frac{D}{D t}\left(\frac{1}{\rho}\right)=0 .
$$


Or

$$
\alpha_{1} \rho_{1} \theta_{1} \frac{D \eta_{1}}{D t}+\alpha_{2} \rho_{2} \theta_{2} \frac{D \eta_{2}}{D t}=\mu\left(p_{1}-p_{2}\right)^{2} \geq 0 .
$$

To satisfy (17) one can choose

$$
\alpha_{a} \rho_{a} \theta_{a} \frac{D \eta_{a}}{D t}=f_{a}=\left(p_{a}-p_{I}\right) \frac{D \alpha_{a}}{D t}, \quad a=1,2 .
$$

where the interface pressure $p_{I}$ can be taken as :

$$
p_{I}=\beta_{2} p_{1}+\beta_{1} p_{2}, \quad \beta_{1}+\beta_{2}=1, \quad \beta_{a} \geq 0 .
$$

Here $\beta_{i}$ are some constants. In particular, the interface pressure $p_{I}$ can be taken as the pressure at the contact discontinuity obtained as the solution of the linearized Riemann problem with initial pressures $p_{1}, p_{2}$ (Saurel, Gavrilyuk and Renaud, 2003). For that case

$$
\beta_{2}=\frac{Z_{2}}{Z_{1}+Z_{2}}, \quad \beta_{1}=\frac{Z_{1}}{Z_{1}+Z_{2}},
$$

where $Z_{a}$ are acoustical impedances of pure phases. With such a choice, the entropies of each phase increase :

$$
\begin{aligned}
& \alpha_{1} \rho_{1} \frac{D \eta_{1}}{D t}=\frac{1}{\theta_{1}}\left(p_{1}-p_{I}\right) \frac{D \alpha_{1}}{D t}=\frac{\mu \beta_{1}}{\theta_{1}}\left(p_{1}-p_{2}\right)^{2} \geq 0, \\
& \alpha_{2} \rho_{2} \frac{D \eta_{2}}{D t}=\frac{1}{\theta_{2}}\left(p_{2}-p_{I}\right) \frac{D \alpha_{2}}{D t}=\frac{\mu \beta_{2}}{\theta_{2}}\left(p_{1}-p_{2}\right)^{2} \geq 0 .
\end{aligned}
$$

Summing these equations we obtain the entropy inequality (16). Finally, the corresponding 1D non-equilibrium model is :

$$
\begin{gathered}
\frac{\partial \rho}{\partial t}+\frac{\partial(\rho u)}{\partial x}=0, \\
\frac{D u}{D t}+\frac{1}{\rho} \frac{\partial p}{\partial x}=0, \\
p=\alpha_{1} p_{1}\left(\frac{Y_{1} \rho}{\alpha_{1}}, \eta_{1}\right)+\alpha_{2} p_{2}\left(\frac{Y_{2} \rho}{\alpha_{2}}, \eta_{2}\right), \\
\frac{D \alpha_{1}}{D t}=\mu\left(p_{1}-p_{2}\right), \\
\frac{D Y_{1}}{D t}=0,
\end{gathered}
$$




$$
\begin{aligned}
\frac{D \eta_{1}}{D t} & =\frac{\mu \beta_{1}}{\alpha_{1} \rho_{1} \theta_{1}}\left(p_{1}-p_{2}\right)^{2}, \\
\frac{D \eta_{2}}{D t} & =\frac{\mu \beta_{2}}{\alpha_{2} \rho_{2} \theta_{2}}\left(p_{1}-p_{2}\right)^{2} .
\end{aligned}
$$

The equations are also hyperbolic, however the characteristic speeds are now : $\lambda_{1,2}=u \pm c_{f}, \lambda_{k}=u, k=3,4,5,6$. Here $c_{f}$ is the "frozen" sound speed :

One can prove that

$$
c_{f}^{2}=Y_{1} c_{1}^{2}+Y_{2} c_{2}^{2}
$$

$$
c_{f}^{2}>c_{w}^{2}
$$

When $\mu \rightarrow \infty$, we recover in this asymptotic limit the equilibrium model (15). The condition (21) is known as Whitham's stability condition (or Whitham's subcharacteristic condition) (Whitham, 1974). The Wood sound speed and the "frozen" sound speed are the velocities of propagation of long and short waves, respectively, for (20).

\section{Bubbly fluids}

In applications (for example, shock wave propagation in bubbly fluids, high velocity impacts etc.) it is necessary to take into account inertia effects. In particular, in such media, the internal energy of a mixture is a function not only of the flow parameters but also their derivatives. Consider a particular case of fluids containing gas bubbles ("bubbly fluids"). For simplicity, we will suppose that the liquid phase is incompressible : only gas bubbles are compressible.

We will use the index "1" for a liquid and the index "2" for a gas. The kinetic energy of an incompressible fluid of density $\rho_{10}=$ const due to single bubble oscillations is (Iordansky (1960), Kogarko (1961), Wijngaarden (1968)) :

$$
2 \pi R^{3} \rho_{10}\left(\frac{d R}{d t}\right)^{2}
$$

where $R$ is the bubble radius. If $N_{2}$ is the number of bubbles per unit volume of the mixture satisfying the conservation law

$$
\frac{\partial N_{2}}{\partial t}+\operatorname{div}\left(N_{2} \mathbf{v}\right)=0
$$

the corresponding energy per unit volume of $N_{2}$ moving bubbles (having the same mean velocity as a surrounding fluid) will be :

$$
2 \pi R^{3} N_{2} \rho_{10}\left(\frac{D R}{D t}\right)^{2}, \quad \frac{D}{D t}=\frac{\partial}{\partial t}+\mathbf{v} \cdot \nabla
$$


The bubble volume fraction is :

$$
\alpha_{2}=\frac{4}{3} \pi R^{3} N_{2}
$$

The Lagrangian of the bubbly fluid can be written in the form :

$$
L=\int_{\mathcal{D}(t)}\left(\frac{\rho|\mathbf{v}|^{2}}{2}+\frac{3}{2} \alpha_{2} \rho_{10}\left(\frac{D R}{D t}\right)^{2}-\rho Y_{2} e_{2}\left(\frac{Y_{2} \rho}{\alpha_{2}}, \eta_{2}\right)\right) d D
$$

We consider the case where the mass concentration of bubbles $Y_{2}=\frac{\alpha_{2} \rho_{2}}{\rho}$, the number of bubbles per unit mass $n_{2}=\frac{N_{2}}{\rho}$ and the gas entropy $\eta_{2}$ are constant. The bubble radius and the gas density can be expressed through the average density $\rho$ as :

$$
\frac{4}{3} \pi R^{3}=\left(\frac{1}{\rho}-\frac{Y_{1}}{\rho_{10}}\right) / n_{2}, \quad \rho_{2}=Y_{2}\left(\frac{1}{\rho}-\frac{Y_{1}}{\rho_{10}}\right)^{-1} .
$$

We rewrite $(22)$ in the following generic form :

$$
L=\int_{\mathcal{D}(t)}\left(\frac{\rho|\mathbf{v}|^{2}}{2}-W\left(\rho, \frac{D \rho}{D t}\right)\right) d D
$$

In our particular case

$$
W\left(\rho, \frac{D \rho}{D t}\right)=\rho\left(Y_{2} e_{2}\left(\rho_{2}, \eta_{2}\right)-2 \pi R^{3} n_{2} \rho_{10}\left(\frac{D R}{D t}\right)^{2}\right)
$$

The usual constraint should be respected :

$$
\frac{\partial \rho}{\partial t}+\operatorname{div}(\rho \mathbf{v})=0
$$

Let us define the variation of such a generic Lagrangian (24). Obviously,

$\delta a$

$$
\begin{gathered}
=\int_{t_{0}}^{t_{1}} \int_{\mathcal{D}(t)}\left(\hat{\delta} \rho\left(\frac{|\mathbf{v}|^{2}}{2}-\frac{\delta W}{\delta \rho}\right)+\rho \mathbf{v} \cdot \hat{\delta} \mathbf{v}-\frac{\partial W}{\partial\left(\frac{D \rho}{D t}\right)} \hat{\delta}\left(\frac{D \rho}{D t}\right)\right) d D d t \\
=\int_{t_{0}}^{t_{1}} \int_{\mathcal{D}(t)}\left(\left(\rho \mathbf{v}-\frac{\partial W}{\partial\left(\frac{D \rho}{D t}\right)} \nabla \rho\right) \cdot \hat{\delta} \mathbf{v}+\hat{\delta} \rho\left(\frac{|\mathbf{v}|^{2}}{2}-\frac{\delta W}{\delta \rho}\right)\right) d D d t
\end{gathered}
$$




$$
\begin{aligned}
& +\int_{t_{0}}^{t_{1}} \int_{\mathcal{D}(t)}\left(-\frac{\partial}{\partial t}\left(\frac{\partial W}{\partial\left(\frac{D \rho}{D t}\right)} \hat{\delta} \rho\right)-\operatorname{div}\left(\frac{\partial W}{\partial\left(\frac{D \rho}{D t}\right)} \mathbf{v} \hat{\delta} \rho\right)\right) d D d t \\
& =\int_{t_{0}}^{t_{1}} \int_{\mathcal{D}(t)}\left(\left(\frac{|\mathbf{v}|^{2}}{2}-\frac{\delta W}{\delta \rho}\right) \hat{\delta} \rho+\left(\rho \mathbf{v}-\frac{\partial W}{\partial\left(\frac{D \rho}{D t}\right)} \nabla \rho\right) \cdot \hat{\delta} \mathbf{v}\right) d D d t \\
& +\int_{t_{0}}^{t_{1}} \int_{\mathcal{D}(t)}\left(-\frac{\partial}{\partial t}\left(\frac{\partial W}{\partial\left(\frac{D \rho}{D t}\right)} \hat{\delta} \rho\right)-\operatorname{div}\left(\frac{\partial W}{\partial\left(\frac{D \rho}{D t}\right)} \mathbf{v} \hat{\delta} \rho\right)\right) d D d t \\
& =\int_{t_{0}}^{t_{1}} \int_{\mathcal{D}(t)}\left(\left(\frac{|\mathbf{v}|^{2}}{2}-\frac{\delta W}{\delta \rho}\right) \hat{\delta} \rho+\left(\rho \mathbf{v}-\frac{\partial W}{\partial\left(\frac{D \rho}{D t}\right)} \nabla \rho\right) \cdot \hat{\delta} \mathbf{v}\right) d D d t .
\end{aligned}
$$

since the conservative terms are vanishing at the boundary $\partial\left(\left[t_{0}, t_{1}\right] \times \mathcal{D}(t)\right)$ . Here we have introduced the classical notation for the variational derivative of $W$ :

$$
\begin{gathered}
\frac{\delta W}{\delta \rho}=\frac{\partial W}{\partial \rho}-\frac{\partial}{\partial t}\left(\frac{\partial W}{\partial\left(\frac{\partial \rho}{\partial t}\right)}\right)-\operatorname{div}\left(\frac{\partial W}{\partial(\nabla \rho)} \mathbf{v}\right) \\
=\frac{\partial W}{\partial \rho}-\frac{\partial}{\partial t}\left(\frac{\partial W}{\partial\left(\frac{D \rho}{D t}\right)}\right)-\operatorname{div}\left(\frac{\partial W}{\partial\left(\frac{D \rho}{D t}\right)} \mathbf{v}\right) \\
=\frac{\partial W}{\partial \rho}-\rho \frac{D}{D t}\left(\frac{1}{\rho} \frac{\partial W}{\partial\left(\frac{D \rho}{D t}\right)}\right)
\end{gathered}
$$

Integrating by parts we obtain

$$
\delta a=\int_{t_{0}}^{t_{1}} \int_{\mathcal{D}(t)} \delta \mathcal{L} d D d t
$$

where

$$
\begin{gathered}
\delta \mathcal{L}=\mathbf{l} \cdot \delta \mathbf{x}, \quad \mathbf{l}=-\rho \frac{D \mathbf{v}}{D t}-\nabla\left(\rho \frac{\delta W}{\delta \rho}-W\right) \\
-\nabla W+\frac{\delta W}{\delta \rho} \nabla \rho+\rho \frac{D}{D t}\left(\frac{1}{\rho} \frac{\partial W}{\partial\left(\frac{D \rho}{D t}\right)} \nabla \rho\right)+\frac{\partial W}{\partial\left(\frac{D \rho}{D t}\right)}\left(\frac{\partial \mathbf{v}}{\partial \mathbf{x}}\right)^{T} \nabla \rho
\end{gathered}
$$


Let us show that the following term vanishes :

$$
-\nabla W+\frac{\delta W}{\delta \rho} \nabla \rho+\rho \frac{D}{D t}\left(\frac{1}{\rho} \frac{\partial W}{\partial\left(\frac{D \rho}{D t}\right)} \nabla \rho\right)+\frac{\partial W}{\partial\left(\frac{D \rho}{D t}\right)}\left(\frac{\partial \mathbf{v}}{\partial \mathbf{x}}\right)^{T} \nabla \rho=0 .
$$

Indeed,

$$
\begin{gathered}
-\nabla W+\frac{\delta W}{\delta \rho} \nabla \rho+\rho \frac{D}{D t}\left(\frac{1}{\rho} \frac{\partial W}{\partial\left(\frac{D \rho}{D t}\right)} \nabla \rho\right)+\frac{\partial W}{\partial\left(\frac{D \rho}{D t}\right)}\left(\frac{\partial \mathbf{v}}{\partial \mathbf{x}}\right)^{T} \nabla \rho \\
=-\frac{\partial W}{\partial \rho} \nabla \rho-\frac{\partial W}{\partial\left(\frac{D \rho}{D t}\right)} \nabla\left(\frac{D \rho}{D t}\right) \\
+\left(\frac{\partial W}{\partial \rho}-\rho \frac{D}{D t}\left(\frac{1}{\rho} \frac{\partial W}{\partial\left(\frac{D \rho}{D t}\right)}\right)\right) \nabla \rho+\rho \frac{D}{D t}\left(\frac{1}{\rho} \frac{\partial W}{\partial\left(\frac{D \rho}{D t}\right)}\right) \nabla \rho \\
+\frac{\partial W}{\partial\left(\frac{D \rho}{D t}\right)} \frac{D \nabla \rho}{D t}+\frac{\partial W}{\partial\left(\frac{D \rho}{D t}\right)}\left(\frac{\partial \mathbf{v}}{\partial \mathbf{x}}\right)^{T} \nabla \rho \\
=-\frac{\partial W}{\partial\left(\frac{D \rho}{D t}\right)} \nabla\left(\frac{D \rho}{D t}\right)+\frac{\partial W}{\partial\left(\frac{D \rho}{D t}\right)} \frac{D \nabla \rho}{D t}+\frac{\partial W}{\partial\left(\frac{D \rho}{D t}\right)}\left(\frac{\partial \mathbf{v}}{\partial \mathbf{x}}\right)^{T} \nabla \rho=0 .
\end{gathered}
$$

Finally, the momentum equation is :

$$
\rho \frac{D \mathbf{v}}{D t}+\nabla\left(\rho \frac{\delta W}{\delta \rho}-W\right)=0 .
$$

The governing equations can also be rewritten in conservative form :

$$
\begin{gathered}
\frac{\partial \rho}{\partial t}+\operatorname{div}(\rho \mathbf{v})=0 \\
\frac{\partial \rho \mathbf{v}}{\partial t}+\operatorname{div}(\rho \mathbf{v} \otimes \mathbf{v})+\nabla p=0 .
\end{gathered}
$$

where the pressure is defined by

$$
p=\rho \frac{\delta W}{\delta \rho}-W
$$


It admits also the energy conservation law :

$$
\frac{\partial}{\partial t}\left(\frac{\rho|\mathbf{v}|^{2}}{2}+E\right)+\operatorname{div}\left(\mathbf{v}\left(\frac{\rho|\mathbf{v}|^{2}}{2}+E+p\right)\right)=0
$$

where

$$
E=W-\frac{D \rho}{D t} \frac{\partial W}{\partial\left(\frac{D \rho}{D t}\right)} .
$$

If $W$ depends only on $\rho$, then $W=E$ and equations (25), (26) coincide with the classical Euler equations of barotropic fluids.

Let us calculate the pressure equation (27) in explicit form for the bubbly fluids. We introduce the specific potential $w$ :

$$
\begin{gathered}
W=\rho w \\
w=Y_{2} e_{2}\left(\rho_{2}, \eta_{2}\right)-2 \pi R^{3} n_{2} \rho_{10}\left(\frac{D R}{D t}\right)^{2} .
\end{gathered}
$$

Obviously,

$$
p=\rho^{2}\left(\frac{\partial w}{\partial \rho}-\frac{D}{D t}\left(\frac{\partial w}{\partial\left(\frac{D \rho}{D t}\right)}\right)\right) .
$$

Consider now $w\left(\rho, \frac{D \rho}{D t}\right)$ as a function of $R$ and $\frac{D R}{D t}$. Then

$$
\begin{gathered}
p=-\frac{1}{4 \pi R^{2} n_{2}}\left(\frac{\partial w}{\partial R}-\frac{D}{D t}\left(\frac{\partial w}{\partial\left(\frac{D R}{D t}\right)}\right)\right) \\
=-\frac{1}{4 \pi R^{2} n_{2}}\left(-p_{2} \frac{3\left(\frac{4 \pi}{3} R^{3} n_{2}\right)^{2}}{\frac{4 \pi}{3} R^{4} n_{2}}-6 \pi R^{2} n_{2} \rho_{10}\left(\frac{D R}{D t}\right)^{2}\right) \\
-\frac{1}{4 \pi R^{2} n_{2}} \frac{D}{D t}\left(4 \pi R^{3} n_{2} \rho_{10}\left(\frac{D R}{D t}\right)\right) \\
=-\frac{1}{4 \pi R^{2} n_{2}}\left(-p_{2} \frac{3\left(\frac{4 \pi}{3} R^{3} n_{2}\right)^{2}}{\frac{4 \pi}{3} R^{4} n_{2}}-6 \pi R^{2} n_{2} \rho_{10}\left(\frac{D R}{D t}\right)^{2}\right. \\
\left.+4 \pi R^{3} n_{2} \rho_{10} \frac{D^{2} R}{D t^{2}}+12 \pi R^{2} n_{2} \rho_{10}\left(\frac{D R}{D t}\right)^{2}\right) \\
=p_{2}+\rho_{10}\left(\frac{3}{2}\left(\frac{D R}{D t}\right)^{2}+R \frac{D^{2} R}{D t^{2}}\right) .
\end{gathered}
$$


The equation

$$
p=p_{2}+\rho_{10}\left(\frac{3}{2}\left(\frac{D R}{D t}\right)^{2}+R \frac{D^{2} R}{D t^{2}}\right)
$$

is known as the Rayleigh-Lamb equation describing oscillations of a single bubble in an infinite fluid. Model (25), (26) and (29) is called in the literature Iordansky-Kogarko-Wijngaarden model (Iordansky (1960), Kogarko (1961), van Wijngaarden (1968)).

\subsection{Dispersive shallow water flows as an analogue of bubbly flows}

Much work has been done in the derivation of relatively simple mathematical models of long non-linear water waves. One of popular models is the Green-Naghdi model obtained in the one-dimensional case by $\mathrm{Su}$ and Gardner (1969) and in the multi-dimensional case by Green, Laws and Naghdi $(1974,1976)$ within the context of a homogeneous one-layer fluid. In the literature, this model is usually called Green-Naghdi model (GN model or GN system). A derivation of the GN model based on the variational formulation of the Euler equations was done by Miles and Salmon (1985) (see also Salmon (1988, 1998)). A mathematical justification of the GN model was done by Makarenko (1986) and Alvarez-Samaniego and Lannes (2008). Solitary wave solutions of the GN model were obtained by Su and Gardner (1969). The linear stability of solitary waves has been proved by $\mathrm{Li}$ (2001). A criterium of stability of shear flows for the GN model was proposed by Gavrilyuk and Teshukov (2004). A wide class of multi-dimensional solutions and approximate solutions of nonlinear multi-dimensional GN model has been found by Gavrilyuk and Teshukov (2001) and Teshukov and Gavrilyuk (2006). Unsteady undular bores were described by El, Grimshaw and Smyth (2006).

It is interesting to note that such a model can be viewed as an analogue of "bubbly fluids" flow model. The Green-Naghdi equations describing shallow water flows over a plane bottom are :

$$
\begin{gathered}
\frac{\partial h}{\partial t}+\operatorname{div}(h \mathbf{v})=0 \\
\frac{\partial h \mathbf{v}}{\partial t}+\operatorname{div}\left(h \mathbf{v} \otimes \mathbf{v}+\left(\frac{g h^{2}}{2}+\frac{h^{2}}{3} \frac{D^{2} h}{D t^{2}}\right) I_{2}\right)=0 .
\end{gathered}
$$

Here $h$ is the position of free surface, $\mathbf{v}$ is the average with respect the vertical direction $2 D$ velocity field, $g$ is the gravity acceleration, and $I_{2}$ is the two-dimensional identity transformation. The Green-Naghdi model is 
formally described with the same type of Lagrangian :

$$
L=\int_{\mathcal{D}(t)}\left(\frac{h|\mathbf{v}|^{2}}{2}-W\left(h, \frac{D h}{D t}\right)\right) d D
$$

where the function $W$ is :

$$
W\left(h, \frac{D h}{D t}\right)=\frac{g h^{2}}{2}-\frac{h}{6}\left(\frac{D h}{D t}\right)^{2} .
$$

Obviously,

$$
p=h \frac{\delta W}{\delta h}-W=\frac{g h^{2}}{2}+\frac{h^{2}}{3} \frac{D^{2} h}{D t^{2}} .
$$

Such an analogy between "bubbly fluids" and dispersive shallow water flows is very useful. Indeed, the parameters of waves propagating in bubbly fluids can not be observed, they can only be measured, while the position of the free surface (playing the role of average density) can be at least easily observed.

\section{Two-velocity one-pressure model}

Consider the case where the averaging velocities of phases are not equal. The Lagrangian of the mixture is :

$$
L=\int_{\mathcal{D}}\left(\sum_{a=1}^{2} \alpha_{a} \rho_{a} \frac{\left|\mathbf{v}_{a}\right|^{2}}{2}-\sum_{a=1}^{2} \alpha_{a} \rho_{a} e_{a}\right) d D
$$

Here $\mathcal{D}$ is not a material volume, this notion has no sense in the case of two velocity continua. We will use the Eulerian variations to define the governing equations. The imposed constraints are

$$
\begin{gathered}
\left(\alpha_{a} \rho_{a}\right)_{t}+\operatorname{div}\left(\alpha_{a} \rho_{a} \mathbf{v}_{a}\right)=0, \\
\left(\alpha_{a} \rho_{a} \eta_{a}\right)_{t}+\operatorname{div}\left(\alpha_{a} \rho_{a} \mathbf{v}_{a} \eta_{a}\right)=0 .
\end{gathered}
$$

Or, in terms of apparent densities $\bar{\rho}_{a}$ :

$$
\begin{gathered}
\left(\bar{\rho}_{a}\right)_{t}+\operatorname{div}\left(\bar{\rho}_{a} \mathbf{v}_{a}\right)=0, \\
\left(\bar{\rho}_{a} \eta_{a}\right)_{t}+\operatorname{div}\left(\bar{\rho}_{a} \mathbf{v}_{a} \eta_{a}\right)=0 .
\end{gathered}
$$

At least three types of independent variations should be considered. The first one is the variation with respect to the volume fraction giving us the equilibrium condition :

$$
p_{1}=p_{2}=p, \quad p=\alpha_{1} p_{1}+\alpha_{2} p_{2} .
$$


The second and the third variations in terms of virtual displacements $\delta \mathbf{x}_{a}$ (which can be introduced in the same way as in the case of one-velocity continua) give us usual expressions of variations of the density, entropy and velocity :

$$
\begin{gathered}
\hat{\delta} \bar{\rho}_{a}=-\operatorname{div}\left(\bar{\rho}_{a} \delta \mathbf{x}_{a}\right), \quad \hat{\delta} \eta_{a}=-\nabla \eta_{a} \cdot \delta \mathbf{x}_{a}, \quad \hat{\delta} \mathbf{v}_{a}=\frac{D_{a} \delta \mathbf{x}_{a}}{D t}-\frac{\partial \mathbf{v}_{a}}{\partial \mathbf{x}} \delta \mathbf{x}_{a} \\
\frac{D_{a}}{D t}=\frac{\partial}{\partial t}+\mathbf{v}_{a} \cdot \nabla
\end{gathered}
$$

The Euler-Lagrange equations correspond to the classical momentum equations for each phase :

$$
\bar{\rho}_{a} \frac{D_{a} \mathbf{v}_{a}}{D t}+\alpha_{a} \nabla p_{a}=0
$$

Taking into account the equilibrium condition they can also be rewritten in non-conservative form :

$$
\begin{aligned}
& \frac{\partial \alpha_{1} \rho_{1} \mathbf{v}_{1}}{\partial t}+\operatorname{div}\left(\alpha_{1} \rho_{1} \mathbf{v}_{1} \otimes \mathbf{v}_{1}+\alpha_{1} p_{1} I\right)=p \nabla \alpha_{1} \\
& \frac{\partial \alpha_{2} \rho_{2} \mathbf{v}_{2}}{\partial t}+\operatorname{div}\left(\alpha_{2} \rho_{2} \mathbf{v}_{2} \otimes \mathbf{v}_{2}+\alpha_{2} p_{2} I\right)=p \nabla \alpha_{2}
\end{aligned}
$$

Summing them we obtain the equation of the total momentum :

$$
\left(\sum_{a=1}^{2} \alpha_{a} \rho_{a} \mathbf{v}_{a}\right)_{t}+\operatorname{div}\left(\sum_{a=1}^{2} \alpha_{a} \rho_{a} \mathbf{v}_{a} \otimes \mathbf{v}_{a}+p I\right)=0
$$

The total energy is :

$$
\begin{gathered}
\left(\sum_{a=1}^{2} \alpha_{a} \rho_{a}\left(e_{a}+\frac{\left|\mathbf{v}_{a}\right|^{2}}{2}\right)\right)_{t} \\
+\operatorname{div}\left(\sum_{a=1}^{2} \alpha_{a} \rho_{a} \mathbf{v}_{a}\left(e_{a}+\frac{\left|\mathbf{v}_{a}\right|^{2}}{2}\right)+\alpha_{a} p_{a} \mathbf{v}_{a}\right)=0 .
\end{gathered}
$$

\subsection{Analysis of the two-velocity one-pressure model}

For simplicity, consider the one-dimensional isentropic case ( $\eta_{a}=$ const ). The equations are :

$$
\left(\alpha_{1} \rho_{1}\right)_{t}+\left(\alpha_{1} \rho_{1} u_{1}\right)_{x}=0
$$




$$
\begin{gathered}
\left(\alpha_{2} \rho_{2}\right)_{t}+\left(\alpha_{2} \rho_{2} u_{2}\right)_{x}=0, \\
\frac{D_{1} u_{1}}{D t}+\frac{p_{x}}{\rho_{1}}=0, \\
\frac{D_{2} u_{2}}{D t}+\frac{p_{x}}{\rho_{2}}=0 .
\end{gathered}
$$

The pressures are then functions of densities:

$$
p_{1}\left(\frac{\bar{\rho}_{1}}{\alpha_{1}}\right)=p_{2}\left(\frac{\bar{\rho}_{2}}{\alpha_{2}}\right)=p .
$$

Using the expression for the differential of the volume fraction obtained from the equilibrium condition (31)

$$
d \alpha_{1}=\frac{\frac{c_{1}^{2}}{\alpha_{1}} d \bar{\rho}_{1}-\frac{c_{2}^{2}}{\alpha_{2}} d \bar{\rho}_{2}}{\frac{c_{1}^{2} \bar{\rho}_{1}}{\alpha_{1}^{2}}+\frac{c_{2}^{2} \bar{\rho}_{2}}{\alpha_{2}^{2}}}=\frac{\frac{c_{1}^{2}}{\alpha_{1}} d \bar{\rho}_{1}-\frac{c_{2}^{2}}{\alpha_{2}} d \bar{\rho}_{2}}{\frac{c_{1}^{2} \rho_{1}}{\alpha_{1}}+\frac{c_{2}^{2} \rho_{2}}{\alpha_{2}}},
$$

and rewriting the equations in variables $\left(\bar{\rho}_{1}, \bar{\rho}_{2}, u_{1}, u_{2}\right)$, one can obtain the following characteristic polynomial for $(30)$ :

$$
\left(\lambda-u_{1}\right)^{2}\left(\lambda-u_{2}\right)^{2}-\frac{\alpha_{2} \rho c_{w}^{2}}{\rho_{2}}\left(\lambda-u_{1}\right)^{2}-\frac{\alpha_{1} \rho c_{w}^{2}}{\rho_{1}}\left(\lambda-u_{2}\right)^{2}=0 .
$$

Transforming it to the form

$$
1=\frac{\alpha_{2} \rho c_{w}^{2}}{\rho_{2}} \frac{1}{\left(\lambda-u_{2}\right)^{2}}+\frac{\alpha_{1} \rho c_{w}^{2}}{\rho_{1}} \frac{1}{\left(\lambda-u_{1}\right)^{2}}
$$

and looking for the minimal value of the right-hand side, one obtain the following criterion of hyperbolicity (existence of four real eigenvalues):

$$
\left(u_{2}-u_{1}\right)^{2}>\rho c_{w}^{2}\left(\left(\frac{\alpha_{1}}{\rho_{1}}\right)^{1 / 3}+\left(\frac{\alpha_{2}}{\rho_{2}}\right)^{1 / 3}\right)^{3} .
$$

However, this model is not hyperbolic for small relative velocity. One can prove that the corresponding volume energy

$$
E=\alpha_{1} \rho_{1} e_{1}+\alpha_{2} \rho_{2} e_{2}=\bar{\rho}_{1} e_{1}+\bar{\rho}_{2} e_{2}
$$

has the following differential :

$$
d E=h_{1} d \bar{\rho}_{1}+h_{2} d \bar{\rho}_{2} .
$$


where $h_{i}, i=1,2$ are the phase enthalpies :

$$
d h_{i}=\frac{d p_{i}}{\rho_{i}}=\frac{d p}{\rho_{i}}
$$

Also,

$$
\begin{gathered}
\frac{\partial^{2} E}{\partial \bar{\rho}_{1}^{2}}=\frac{\rho c_{w}^{2}}{\rho_{1}^{2}} \\
\frac{\partial^{2} E}{\partial \bar{\rho}_{2}^{2}}=\frac{\partial h_{2}}{\partial \bar{\rho}_{2}}=\frac{c_{2}^{2}}{\rho_{2}}\left(\frac{1}{\alpha_{2}}+\frac{\bar{\rho}_{2}}{\alpha_{2}^{2}} \frac{\partial \alpha_{1}}{\partial \bar{\rho}_{2}}\right)=\frac{\rho c_{w}^{2}}{\rho_{2}^{2}} \\
\frac{\partial^{2} E}{\partial \bar{\rho}_{1} \partial \bar{\rho}_{2}}=\frac{\rho c_{w}^{2}}{\rho_{1} \rho_{2}} .
\end{gathered}
$$

In particular this implies that the corresponding Hessian matrix is degenerate:

$$
\operatorname{det}\left(\begin{array}{cc}
\frac{\partial^{2} E}{\partial \bar{\rho}_{1}^{2}} & \frac{\partial^{2} E}{\partial \bar{\rho}_{1} \partial \bar{\rho}_{2}} \\
\frac{\partial^{2} E}{\partial \bar{\rho}_{1} \partial \bar{\rho}_{2}} & \frac{\partial^{2} E}{\partial \bar{\rho}_{2}^{2}}
\end{array}\right)=0 .
$$

In mechanics, the non-convexity of the internal energy shows up nonstability of the mechanical system. In our case, the degeneracy of the internal energy is responsible for the non-hyperbolicity of the governing equations and, as a consequence, the ill-posedness of the corresponding Cauchy problem.

\section{Two-velocity two-pressure model}

The following non-equilibrium model obtained by adding relaxation terms in the pressure equilibrium model is called Baer-Nunziato model (1986) (or $\mathrm{BN}$ model) :

$$
\begin{gathered}
\frac{\partial \alpha_{1} \rho_{1}}{\partial t}+\operatorname{div}\left(\alpha_{1} \rho_{1} \mathbf{v}_{1}\right)=0 \\
\frac{\partial \alpha_{2} \rho_{2}}{\partial t}+\operatorname{div}\left(\alpha_{2} \rho_{2} \mathbf{v}_{2}\right)=0 \\
\frac{\partial \alpha_{1} \rho_{1} \mathbf{v}_{1}}{\partial t}+\operatorname{div}\left(\alpha_{1} \rho_{1} \mathbf{v}_{1} \otimes \mathbf{v}_{1}+\alpha_{1} p_{1} I\right)=p_{I} \nabla \alpha_{1}+\lambda\left(\mathbf{v}_{2}-\mathbf{v}_{1}\right), \\
\frac{\partial \alpha_{2} \rho_{2} \mathbf{v}_{2}}{\partial t}+\operatorname{div}\left(\alpha_{2} \rho_{2} \mathbf{v}_{2} \otimes \mathbf{v}_{2}+\alpha_{2} p_{2} I\right)=p_{I} \nabla \alpha_{2}-\lambda\left(\mathbf{v}_{2}-\mathbf{v}_{1}\right), \\
\frac{\partial}{\partial t}\left(\alpha_{1} \rho_{1}\left(\frac{\left|\mathbf{v}_{1}\right|^{2}}{2}+e_{1}\right)\right)+\operatorname{div}\left(\alpha_{1} \rho_{1} \mathbf{v}_{1}\left(\frac{\left|\mathbf{v}_{1}\right|^{2}}{2}+e_{1}\right)+\alpha_{1} p_{1} \mathbf{v}_{1}\right)=
\end{gathered}
$$




$$
\begin{gathered}
=-p_{I} \frac{\partial \alpha_{1}}{\partial t}+\lambda \mathbf{v}_{1} \cdot\left(\mathbf{v}_{2}-\mathbf{v}_{1}\right) \\
\frac{\partial}{\partial t}\left(\alpha_{2} \rho_{2}\left(\frac{\left|\mathbf{v}_{2}\right|^{2}}{2}+e_{2}\right)\right)+\operatorname{div}\left(\alpha_{2} \rho_{2} \mathbf{v}_{2}\left(\frac{\left|\mathbf{v}_{2}\right|^{2}}{2}+e_{2}\right)+\alpha_{2} p_{2} \mathbf{v}_{2}\right)= \\
=-p_{I} \frac{\partial \alpha_{2}}{\partial t}-\lambda \mathbf{v}_{1} \cdot\left(\mathbf{v}_{2}-\mathbf{v}_{1}\right) \\
\frac{D_{I} \alpha_{1}}{D t}=\mu\left(p_{1}-p_{2}\right), \quad \frac{D_{I}}{D t}=\frac{\partial}{\partial t}+\mathbf{v}_{I} \cdot \nabla .
\end{gathered}
$$

Here $p_{I}$ is the "interface" pressure, $\mathbf{v}_{I}$ is the "interface" velocity, and $\lambda$ is the friction coefficient. In the classical BN model the following duality is supposed $p_{I}=p_{2}$ and $\mathbf{v}_{I}=\mathbf{v}_{1}$, or $p_{I}=p_{1}$ and $\mathbf{v}_{I}=\mathbf{v}_{2}$. The right-hand side in the momentum equations is the sum of "nozzling terms" $p_{I} \nabla \alpha_{a}$ (the word "nozzling" is to underline the analogy between these equations and those describing gas flow in the duct of variable cross section) and Stokes type terms $\lambda\left(\mathbf{v}_{2}-\mathbf{v}_{1}\right)$. Other choice of "interface" variables is possible (see, for example, Saurel, Gavrilyuk and Renaud, 2003). Such a system of equations satisfies the entropy inequality and has only real eigenvalues.

\section{Equilibrium diffuse interface model of solid-fluid interactions}

Pioneering works by Karni (1994), Abgrall (1996) and Saurel and Abgrall (1999) have shown the attractivity the diffuse interface approach for modelling interfaces between ideal fluids having different thermodynamic characteristics. In such an approach the interface between different materials is considered as a diffusion zone : it is a mixture of two components where the volume fraction of the phases varies continuously from zero to one. To assure the non-degeneracy of the governing equations, one also supposes that even in the bulk (where, formally, we have only pure phases ) a negligible quantity of the other phase is present. Hence, the interface can be viewed as an effective fluid mixture. We will generalize this approach to the case of solid-fluid interfaces by using Hamilton's principle (Gavrilyuk et al. (2008), Favrie et al. (2009)).

Consider the following Lagrangian for a solid-fluid mixture :

$$
L=\int_{\Omega_{t}} \rho\left(\frac{\mathbf{v} \cdot \mathbf{v}}{2}-e\right) d \Omega
$$

where the average density and the average volume energy are defined as

$$
\rho=\alpha_{s} \rho_{s}+\alpha_{g} \rho_{g}, \quad \rho e=\alpha_{s} \rho_{s} e_{s}+\alpha_{g} \rho_{g} e_{g} .
$$


The indices "s" and "g" mean "solid" and "gas", respectively. The mass conservation laws can be rewritten in terms of the mass fractions $Y_{s}$ and $Y_{g}$

$$
\frac{D Y_{s}}{D t}=0, \quad \frac{D Y_{g}}{D t}=0, \quad Y_{s}=\frac{\alpha_{s} \rho_{s}}{\rho}, \quad Y_{g}=\frac{\alpha_{g} \rho_{g}}{\rho}, \quad Y_{s}+Y_{g}=1
$$

Also, the entropies of each phase are conserved :

$$
\frac{D \eta_{s}}{D t}=0, \quad \frac{D \eta_{g}}{D t}=0
$$

The energy of the solid phase $e_{s}$ is a function of the entropy $\eta_{s}$ and the mixture deformation tensor

$$
G=\sum_{\beta=1}^{3} \mathbf{E}^{\beta} \otimes \mathbf{E}^{\beta}
$$

where

$$
\mathbf{E}^{\beta}=\nabla X^{\beta}
$$

and $\mathbf{X}=\left(X^{\beta}\right)$ are the Lagrangian coordinates of a mixture particle. The mixture density is related to $G$ by the formula :

$$
\rho(t, \mathbf{X})=\rho_{0}(\mathbf{X})|G|^{1 / 2}
$$

We use here capital letters $\mathbf{E}^{\beta}$ to distinguish the mixture case from the pure solid one. The gas energy $e_{g}$ is a function of the gas density $\rho_{g}$ and the gas entropy $\eta_{s}$.

To apply the Hamilton principle we find the variations of $\rho, \mathbf{v}, Y_{s}, Y_{g}, \eta_{g}$, $\eta_{s}$ as functions of virtual displacements $\delta \mathbf{x}$ at fixed Lagrangian coordinates :

$$
\begin{aligned}
& \tilde{\delta} \rho=-\rho \operatorname{div}(\delta \mathbf{x}), \quad \tilde{\delta} \mathbf{v}=\frac{\partial \delta \mathbf{x}}{\partial t}, \quad \tilde{\delta} \eta_{i}=0, \quad \tilde{\delta} Y_{i}=0 . \\
& \tilde{\delta} \mathbf{E}^{\beta}=-\left(\frac{\partial \delta \mathbf{x}}{\partial \mathbf{x}}\right)^{T} \mathbf{E}^{\beta} .
\end{aligned}
$$

As usually, the symbol $\tilde{\delta}$ means the variation at fixed Lagrangian coordinates. The last formula giving the variation of $\mathbf{E}^{\beta}$ can be found in the following way. There exist potentials $X^{\beta}, \beta=1,2,3$ (the mixture Lagrangian coordinates) such that

$$
\frac{D X^{\beta}}{D t}=0
$$


It follows from the definition of $X^{\beta}$ that its Lagrangian variation is zero :

$$
\tilde{\delta} X^{\beta}=0 .
$$

Since

$$
\tilde{\delta} F^{-1}=-F^{-1} \tilde{\delta} F F^{-1}=-F^{-1} \frac{\partial \delta \mathbf{x}}{\partial \mathbf{X}} F^{-1}=-F^{-1} \frac{\partial \delta \mathbf{x}}{\partial \mathbf{x}}
$$

we get from the definition of $\mathbf{E}^{\beta}$ :

$$
\begin{aligned}
\tilde{\delta} \mathbf{E}^{\beta}= & \tilde{\delta} \nabla X^{\beta}=\tilde{\delta}\left(F^{-T} \nabla_{\mathbf{X}} X^{\beta}\right)=-\left(F^{-1} \frac{\partial \delta \mathbf{x}}{\partial \mathbf{x}}\right)^{T} \nabla_{\mathbf{X}} X^{\beta} \\
& =-\left(\frac{\partial \delta \mathbf{x}}{\partial \mathbf{x}}\right)^{T} F^{-T} \nabla_{\mathbf{X}} X^{\beta}=-\left(\frac{\partial \delta \mathbf{x}}{\partial \mathbf{x}}\right)^{T} \mathbf{E}^{\beta} .
\end{aligned}
$$

In particular, the variation of $G=\sum_{\beta=1}^{3} \mathbf{E}^{\beta} \otimes \mathbf{E}^{\beta}$ is given by :

$$
\tilde{\delta} G=\tilde{\delta}\left(\sum_{\beta=1}^{3} \mathbf{E}^{\beta} \otimes \mathbf{E}^{\beta}\right)=-\left(\frac{\partial \delta \mathbf{x}}{\partial \mathbf{x}}\right)^{T} G-G\left(\frac{\partial \delta \mathbf{x}}{\partial \mathbf{x}}\right) .
$$

The energy of the solid is taken in separable form

$$
e_{s}=e_{s}^{h}\left(\rho_{s}, \eta_{s}\right)+e_{s}^{e}(g), \quad g=\frac{G}{|G|^{1 / 3}} .
$$

Its variation is :

$$
\begin{gathered}
\tilde{\delta} e_{s}=\tilde{\delta} e_{s}^{h}\left(\frac{Y_{s} \rho}{\alpha_{s}}, \eta_{s}\right)+\tilde{\delta} e_{s}^{e}(g) \\
=\frac{\partial e_{s}^{h}}{\partial \rho_{s}} \tilde{\delta}\left(\frac{Y_{s} \rho}{\alpha_{s}}\right)+\operatorname{tr}\left(\frac{\partial e_{s}^{e}}{\partial G} \tilde{\delta} G\right)+\theta_{s} \tilde{\delta} \eta_{s} \\
=\frac{\rho Y_{s}}{\alpha_{s}} \frac{\partial e_{s}^{h}}{\partial \rho_{s}}\left(\frac{\tilde{\delta} \rho}{\rho}-\frac{\tilde{\delta} \alpha_{s}}{\alpha_{s}}\right)+\operatorname{tr}\left(\frac{\partial e_{s}^{e}}{\partial G} \tilde{\delta} G\right) \\
=\frac{p_{s}}{\rho_{s}}\left(\frac{\tilde{\delta} \rho}{\rho}-\frac{\tilde{\delta} \alpha_{s}}{\alpha_{s}}\right)+\operatorname{tr}\left(\frac{\partial e_{s}^{e}}{\partial G} \tilde{\delta} G\right) \\
=\frac{p_{s}}{\rho_{s}}\left(\frac{\tilde{\delta} \rho}{\rho}-\frac{\tilde{\delta} \alpha_{s}}{\alpha_{s}}\right)-\frac{1}{2 \rho_{s}} \operatorname{tr}\left(S_{s} G^{-1} \tilde{\delta} G\right)
\end{gathered}
$$




$$
\begin{gathered}
=\frac{p_{s}}{\rho_{s}}\left(\frac{\tilde{\delta} \rho}{\rho}-\frac{\tilde{\delta} \alpha_{s}}{\alpha_{s}}\right)+\frac{1}{\rho_{s}} \operatorname{tr}\left(S_{s} \frac{\partial \delta \mathbf{x}}{\partial \mathbf{x}}\right) \\
=\frac{p_{s}}{\rho_{s}}\left(\frac{\tilde{\delta} \rho}{\rho}-\frac{\tilde{\delta} \alpha_{s}}{\alpha_{s}}\right)+\frac{1}{\rho_{s}} \operatorname{tr}\left(S_{s} \frac{\partial \delta \mathbf{x}}{\partial \mathbf{x}}\right) \\
=\frac{p_{s}}{\rho_{s}}\left(-\operatorname{div}(\delta \mathbf{x})-\frac{\tilde{\delta} \alpha_{s}}{\alpha_{s}}\right)+\frac{1}{\rho_{s}} \operatorname{tr}\left(S_{s} \frac{\partial \delta \mathbf{x}}{\partial \mathbf{x}}\right) \\
=\frac{p_{s}}{\rho_{s}}\left(-\frac{\tilde{\delta} \alpha_{s}}{\alpha_{s}}\right)+\frac{1}{\rho_{s}} \operatorname{tr}\left(\left(-p_{s} I+S_{s}\right) \frac{\partial \delta \mathbf{x}}{\partial \mathbf{x}}\right) \\
=-\frac{1}{\rho_{s}} \operatorname{tr}\left(\sigma_{s} \frac{\partial \delta \mathbf{x}}{\partial \mathbf{x}}\right)-\frac{p_{s}}{\alpha_{s} \rho_{s}} \tilde{\delta} \alpha_{s} .
\end{gathered}
$$

Here

$$
S_{s}=-2 \rho_{s} \frac{\partial e_{s}^{e}}{\partial G} G
$$

is the deviatoric part of the stress tensor

$$
\sigma_{s}=-p_{s} I+S_{s}, \quad \operatorname{tr}\left(S_{s}\right)=0 .
$$

Finally,

$$
\tilde{\delta} e_{s}=-\frac{1}{\rho_{s}} \operatorname{tr}\left(\sigma_{s} \frac{\partial \delta \mathbf{x}}{\partial \mathbf{x}}\right)-\frac{p_{s}}{\alpha_{s} \rho_{s}} \tilde{\delta} \alpha_{s}
$$

Analogous considerations give a simpler variation for the gas phase :

$$
\tilde{\delta} e_{g}=-\frac{1}{\rho_{g}} \operatorname{tr}\left(\sigma_{g} \frac{\partial \delta \mathbf{x}}{\partial \mathbf{x}}\right)-\frac{p_{g}}{\alpha_{g} \rho_{g}} \tilde{\delta} \alpha_{s}=\frac{p_{g}}{\rho_{g}} \operatorname{div} \delta \mathbf{x}-\frac{p_{g}}{\alpha_{g} \rho_{g}} \tilde{\delta} \alpha_{g}
$$

Now, we are ready to take the variation of the Hamilton action. We use formulas (33), (35), (36), the mass conservation law $\rho d \Omega=\rho_{0}(\mathbf{X}) d \Omega_{0}$ to present the variation of $a$ in the form :

$$
\begin{gathered}
0=\delta a=\delta \int_{t_{1}}^{t_{2}} \int_{\Omega_{t}} \rho\left(\frac{\mathbf{v} \cdot \mathbf{v}}{2}-e\right) d \Omega d t \\
=\delta \int_{t_{1}}^{t_{2}} \int_{\Omega_{0}} \rho_{0}(\mathbf{X})\left(\frac{\mathbf{v} \cdot \mathbf{v}}{2}-e\right) d \Omega_{0} d t
\end{gathered}
$$




$$
\begin{gathered}
=\int_{t_{1}}^{t_{2}} \int_{\Omega_{0}} \rho_{0}(\mathbf{X})(\tilde{\delta} \mathbf{v} \cdot \mathbf{v}-\tilde{\delta} e) d \Omega_{0} d t \\
=\int_{t_{1}}^{t_{2}} \int_{\Omega_{0}} \rho_{0}(\mathbf{X})\left(\frac{\partial \delta \mathbf{x}}{\partial t} \cdot \mathbf{v}+Y_{s}\left(\frac{1}{\rho_{s}} \operatorname{tr}\left(\sigma_{s} \frac{\partial \delta \mathbf{x}}{\partial \mathbf{x}}\right)+\frac{p_{s}}{\alpha_{s} \rho_{s}} \tilde{\delta} \alpha_{s}\right)\right) d \Omega_{0} d t \\
-\int_{t_{1}}^{t_{\Omega_{0}}} \rho_{0}(\mathbf{X})\left(Y_{g}\left(\frac{p_{g}}{\rho_{g}} \operatorname{div} \delta \mathbf{x}-\frac{p_{g}}{\alpha_{g} \rho_{g}} \tilde{\delta} \alpha_{g}\right)\right) d \Omega_{0} d t \\
=\int_{t_{1}}^{t_{2}} \int_{\Omega_{0}} \rho_{0}(\mathbf{X})\left(\frac{\partial \delta \mathbf{x}}{\partial t} \cdot \mathbf{v}\right) d \Omega_{0} d t \\
+\int_{t_{1}}^{t_{2}} \frac{\rho_{\Omega_{0}}}{\rho}\left(\operatorname{tr}\left(\left(\alpha_{s} \sigma_{s}-\alpha_{g} p_{g}\right) \frac{\partial \delta \mathbf{x}}{\partial \mathbf{x}}\right)+p_{s} \tilde{\delta} \alpha_{s}+p_{g} \tilde{\delta} \alpha_{g}\right) d \Omega_{0} d t
\end{gathered}
$$

Since $\tilde{\delta} \alpha_{s}+\tilde{\delta} \alpha_{g}=0$, it implies the equilibrium condition :

$$
p_{s}-p_{g}=0 .
$$

Here

$$
p_{s}=-\frac{1}{3} \operatorname{tr}\left(\sigma_{s}\right), \quad p_{g}=-\frac{1}{3} \operatorname{tr}\left(\sigma_{g}\right) .
$$

Turning back to the Eulerian coordinates we transform the last variation to the following one :

$$
0=\delta a=\int_{t_{1}}^{t_{2}} \int_{\Omega_{t}}\left(\frac{D \delta \mathbf{x}}{D t} \cdot \rho \mathbf{v}+\operatorname{tr}\left(\left(\alpha_{s} \sigma_{s}-\alpha_{g} p_{g}\right) \frac{\partial \delta \mathbf{x}}{\partial \mathbf{x}}\right)\right) d \Omega_{t} d t
$$

Integrating by parts and taking into account the fact that $\delta \mathbf{x}$ is vanishing at the boundary $\Omega_{t} \times\left[t_{1}, t_{2}\right]$ we obtain

$$
0=\delta a=-\int_{t_{1}}^{t_{2}} \int_{\Omega_{t}}\left(\frac{\partial \rho \mathbf{v}}{\partial t}+\operatorname{div}\left(\rho \mathbf{v} \otimes \mathbf{v}-\left(\alpha_{s} \sigma_{s}+\alpha_{g} \sigma_{g}\right)\right)\right) \cdot \delta \mathbf{x} d \Omega d t
$$

for any $\delta \mathbf{x}$ vanishing at the boundary $\partial\left(\Omega_{t} \times\left[t_{1}, t_{2}\right]\right)$. It implies the momentum equation :

$$
\frac{\partial \rho \mathbf{v}}{\partial t}+\operatorname{div}\left(\rho \mathbf{v} \otimes \mathbf{v}-\left(\alpha_{s} \sigma_{s}+\alpha_{g} \sigma_{g}\right)\right)=0 .
$$


The entropy equations

$$
\frac{D \eta_{s}}{D t}=0, \quad \frac{D \eta_{g}}{D t}=0
$$

complemented by the mass and momentum balance laws imply the mixture energy conservation law:

$$
\frac{\partial}{\partial t}\left(\rho\left(e+\frac{\mathbf{v} \cdot \mathbf{v}}{2}\right)\right)+\operatorname{div}\left(\rho \mathbf{v}\left(e+\frac{\mathbf{v} \cdot \mathbf{v}}{2}\right)-\left(\alpha_{s} \sigma_{s}+\alpha_{g} \sigma_{g}\right) \mathbf{v}\right)=0 .
$$

Finally, the equilibrium solid-gas governing equations are

$$
\begin{gathered}
\frac{\partial \mathbf{E}^{\beta}}{\partial t}+\nabla\left(\mathbf{E}^{\beta} \cdot \mathbf{v}\right)=-\operatorname{rot} \mathbf{E}^{\beta} \wedge \mathbf{v}, \\
\operatorname{rot} \mathbf{E}^{\beta}=0, \\
\frac{\partial\left(\alpha_{g} \rho_{g}\right)}{\partial t}+\operatorname{div}\left(\alpha_{g} \rho_{g} \mathbf{v}\right)=0, \\
\frac{\partial \rho \mathbf{v}}{\partial t}+\operatorname{div}\left(\rho \mathbf{v} \otimes \mathbf{v}-\left(\alpha_{s} \sigma_{s}+\alpha_{g} \sigma_{g}\right)\right)=0, \\
\frac{D \eta_{s}}{D t}=0, \quad \frac{D \eta_{g}}{D t}=0, \\
p_{s}=p_{g} .
\end{gathered}
$$

Equations (40) admit the energy conservation law (39).

\section{Non-equilibrium diffuse interface model of solid-fluid interactions}

For numerical reasons it is preferable to use a non-equilibrium model where the algebraic equation for the volume fraction $p_{s}-p_{g}=0$ is replaced by a differential equation. This procedure will be done in the same way as for fluid-fluid mixtures. The relaxation equation will be :

$$
p_{s}-p_{g}=\frac{1}{\mu} \frac{D \alpha_{s}}{D t}
$$

Now, we postulate the mixture energy equation :

$$
\frac{\partial}{\partial t}\left(\rho\left(e+\frac{\mathbf{v} \cdot \mathbf{v}}{2}\right)\right)+\operatorname{div}\left(\rho \mathbf{v}\left(e+\frac{\mathbf{v} \cdot \mathbf{v}}{2}\right)-\left(\alpha_{s} \sigma_{s}+\alpha_{g} \sigma_{g}\right) \mathbf{v}\right)=0 .
$$

As in the case of fluid-fluid mixtures, we can transform the energy equation (41) to the following one :

$$
\alpha_{s} \rho_{s} \theta_{s} \frac{D \eta_{s}}{D t}+\alpha_{g} \rho_{g} \theta_{g} \frac{D \eta_{g}}{D t}=\left(p_{s}-p_{g}\right) \frac{D \alpha_{s}}{D t} .
$$


Indeed,

$$
\begin{gathered}
0=\frac{\partial}{\partial t}\left(\rho\left(e+\frac{\mathbf{v} \cdot \mathbf{v}}{2}\right)\right)+\operatorname{div}\left(\rho \mathbf{v}\left(e+\frac{\mathbf{v} \cdot \mathbf{v}}{2}\right)-\left(\alpha_{s} \sigma_{s}+\alpha_{g} \sigma_{g}\right) \mathbf{v}\right) \\
=\rho \frac{D e}{D t}-\operatorname{tr}\left(\left(\alpha_{s} \sigma_{s}+\alpha_{g} \sigma_{g}\right) \frac{\partial \mathbf{v}}{\partial \mathbf{x}}\right) \\
=\rho\left(Y_{s} \frac{\partial e_{s}^{h}}{\partial \rho_{s}} \frac{D \rho_{s}}{D t}+Y_{s} \operatorname{tr}\left(\frac{\partial e_{s}^{e}}{\partial G} \frac{D G}{D t}\right)+Y_{s} \frac{\partial e_{s}}{\partial \eta_{s}} \frac{D \eta_{s}}{D t}\right. \\
\left.+Y_{g} \frac{\partial e_{g}}{\partial \rho_{g}} \frac{D \rho_{g}}{D t}+Y_{g} \frac{\partial e_{g}}{\partial \eta_{g}} \frac{D \eta_{g}}{D t}\right)-\operatorname{tr}\left(\left(\alpha_{s} \sigma_{s}-\alpha_{g} p_{g} I\right) \frac{\partial \mathbf{v}}{\partial \mathbf{x}}\right) \\
=\alpha_{s} p_{s}\left(-\frac{1}{\alpha_{s}} \frac{D \alpha_{s}}{D t}-\operatorname{div} \mathbf{v}\right)+\operatorname{tr}\left(\alpha_{s} S_{s} \frac{\partial \mathbf{v}}{\partial \mathbf{x}}\right)+\rho Y_{s} \frac{\partial e_{s}}{\partial \eta_{s}} \frac{D \eta_{s}}{D t} \\
+\alpha_{g} p_{g}\left(-\frac{1}{\alpha_{g}} \frac{D \alpha_{g}}{D t}-\operatorname{div} \mathbf{v}\right)+\rho Y_{g} \frac{\partial e_{g}}{\partial \eta_{g}} \frac{D \eta_{g}}{D t}-\operatorname{tr}\left(\left(\alpha_{s} \sigma_{s}-\alpha_{g} p_{g} I\right) \frac{\partial \mathbf{v}}{\partial \mathbf{x}}\right) \\
=\rho\left(Y_{s} \theta_{s} \frac{D \eta_{s}}{D t}+Y_{g} \theta_{g} \frac{D \eta_{g}}{D t}\right)-\left(p_{s}-p_{g}\right) \frac{D \alpha_{s}}{D t} .
\end{gathered}
$$

As in the case of fluid mixtures, the same equations (18) for the entropy production can be used :

$$
\alpha_{s} \rho_{s} \theta_{s} \frac{D \eta_{s}}{D t}=\left(p_{s}-p_{I}\right) \frac{D \alpha_{s}}{D t}, \quad \alpha_{g} \rho_{g} \theta_{g} \frac{D \eta_{g}}{D t}=\left(p_{g}-p_{I}\right) \frac{D \alpha_{s}}{D t} .
$$

Obviously, they are compatible with (42). Finally, the non-equilibrium model is :

$$
\begin{gathered}
\frac{\partial\left(\mathbf{E}^{\beta}\right)}{\partial t}+\nabla\left(\mathbf{E}^{\beta} \cdot \mathbf{v}\right)=-\operatorname{rot} \mathbf{E}^{\beta} \wedge \mathbf{v}, \\
\frac{\partial\left(\alpha_{g} \rho_{g}\right)}{\partial t}+\operatorname{div}\left(\alpha_{g} \rho_{g} \mathbf{v}\right)=0, \\
\frac{\partial \rho \mathbf{v}}{\partial t}+\operatorname{div}\left(\rho \mathbf{v} \otimes \mathbf{v}-\left(\alpha_{s} \sigma_{s}+\alpha_{g} \sigma_{g}\right)\right)=0, \\
\alpha_{s} \rho_{s} \theta_{s} \frac{D \eta_{s}}{D t}=\left(p_{s}-p_{I}\right) \frac{D \alpha_{s}}{D t}, \quad \alpha_{g} \rho_{g} \theta_{g} \frac{D \eta_{g}}{D t}=-\left(p_{g}-p_{I}\right) \frac{D \alpha_{s}}{D t}, \\
\frac{D \alpha_{s}}{D t}=\mu\left(p_{s}-p_{g}\right), \quad \mu>0 .
\end{gathered}
$$


The geometric equations (43a) and the entropy equations (43d) can also be rewritten in different forms which are more convenient for numerical computations :

$$
\begin{gathered}
\frac{\partial \mathbf{E}^{\beta}}{\partial t}+\frac{\partial \mathbf{E}^{\beta}}{\partial \mathbf{x}} \mathbf{v}+\left(\frac{\partial \mathbf{v}}{\partial \mathbf{x}}\right)^{T} \mathbf{E}^{\beta}=0 \\
\frac{\partial}{\partial t}\left(\alpha_{s} \rho_{s} e_{s}\right)+\operatorname{div}\left(\alpha_{s} \rho_{s} e_{s} \mathbf{v}\right)-\alpha_{s} \operatorname{tr}\left(\sigma_{s} \frac{\partial \mathbf{v}}{\partial \mathbf{x}}\right)=-p_{I} \mu\left(p_{s}-p_{g}\right) \\
\frac{\partial}{\partial t}\left(\alpha_{g} \rho_{g} e_{g}\right)+\operatorname{div}\left(\alpha_{g} \rho_{g} e_{g} \mathbf{v}\right)-\alpha_{g} \operatorname{tr}\left(\sigma_{g} \frac{\partial \mathbf{v}}{\partial \mathbf{x}}\right)=-p_{I} \mu\left(p_{g}-p_{s}\right)
\end{gathered}
$$

\subsection{Hyperbolicity of the non-equilibrium model}

Let us show that the non-equilibrium model (43) is hyperbolic. We present here the proof of hyperbolicity in the one-dimensional case. We denote $\mathbf{v}=(u, v, w)^{T}$. Let $\mathbf{E}^{\beta}=\left(A^{(\beta)}, B^{(\beta)}, C^{(\beta)}\right)$. Relation $\operatorname{rot} \mathbf{E}^{\beta}=0$ implies that only components $A^{(\beta)}$ vary, the other components are constant. We can always suppose that initially the vectors $\mathbf{E}^{\beta}$ coincide with the Cartesian basis. Hence, $B^{(2)}=C^{(3)}=1, B^{(1)}=B^{(3)}=C^{(1)}=C^{(2)}=0$. The matrix $G$ becomes :

$$
G=\left(\begin{array}{ccc}
\left(A^{(1)}\right)^{2}+\left(A^{(2)}\right)^{2}+\left(A^{(3)}\right)^{2} & A^{(2)} & A^{(3)} \\
A^{(2)} & 1 & 0 \\
A^{(3)} & 0 & 1
\end{array}\right)
$$

Obviously,

$$
\begin{gathered}
|G|=\left(A^{(1)}\right)^{2} \\
J_{1}=\operatorname{tr}(G)=\left(A^{(1)}\right)^{2}+\left(A^{(2)}\right)^{2}+\left(A^{(3)}\right)^{2}+2 \\
J_{2}=\operatorname{tr}\left(G^{2}\right)=\left(\left(A^{(1)}\right)^{2}+\left(A^{(2)}\right)^{2}+\left(A^{(3)}\right)^{2}\right)^{2} \\
+2\left(\left(A^{(2)}\right)^{2}+\left(A^{(3)}\right)^{2}\right)+2 .
\end{gathered}
$$

We remark that the invariants $J_{1}$ and $J_{2}$ depend only on $A^{(1)}$ and $Z=$ 
$\sqrt{\left(A^{(2)}\right)^{2}+\left(A^{(3)}\right)^{2}}$. The one-dimensional governing equations become :

$$
\begin{gathered}
\frac{\partial A^{(1)}}{\partial t}+\frac{\partial\left(A^{(1)} u\right)}{\partial x}=0, \\
\frac{\partial A^{(2)}}{\partial t}+\frac{\partial\left(A^{(2)} u+v\right)}{\partial x}=0, \\
\frac{\partial A^{(3)}}{\partial t}+\frac{\partial\left(A^{(3)} u+w\right)}{\partial x}=0, \\
\frac{\partial \rho}{\partial t}+\frac{\partial(\rho u)}{\partial x}=0, \\
\frac{\partial \rho Y_{g}}{\partial t}+\frac{\partial\left(\rho Y_{g} u\right)}{\partial x}=0, \\
\frac{\partial\left(\rho u^{2}-\alpha_{s} \sigma_{s 11}-\alpha_{g} \sigma_{g 11}\right)}{\partial x}=0, \\
\frac{\partial \rho v}{\partial t}+\frac{\partial\left(\rho u v-\alpha_{s} \sigma_{s 12}-\alpha_{g} \sigma_{g 12}\right)}{\partial x}=0, \\
\frac{\partial \rho \alpha_{g}}{\partial t}+\frac{\partial\left(\rho u w-\alpha_{s} \sigma_{s 13}-\alpha_{g} \sigma_{g 13}\right)}{\partial x}=0, \\
\frac{\partial\left(\rho \eta_{s}\right)}{\partial t}+\operatorname{div}\left(\rho \eta_{s} \mathbf{v}\right)=\frac{\left(p_{s}-p_{I}\right)}{Y_{s} \theta_{s}} \frac{D \alpha_{s}}{D t}, \\
\frac{\partial\left(\rho \eta_{g}\right)}{\partial t}+\operatorname{div}\left(\rho \eta_{g} \mathbf{v}\right)=\frac{\left(p_{g}-p_{I}\right)}{Y_{g} \theta_{g}} \frac{D \alpha_{g}}{D t} .
\end{gathered}
$$

System (45) admits the energy conservation law :

$$
\begin{gathered}
\frac{\partial}{\partial t}\left(\rho\left(e+\frac{u^{2}+v^{2}+w^{2}}{2}\right)\right)+\frac{\partial}{\partial x}\left(\rho u\left(e+\frac{u^{2}+v^{2}+w^{2}}{2}\right)\right. \\
\left.-\sigma_{11} u-\sigma_{12} v-\sigma_{13} w\right)=0,
\end{gathered}
$$

where

$$
\begin{gathered}
\sigma_{i j}=\alpha_{s} \sigma_{s i j}+\alpha_{g} \sigma_{g i j}, \\
\rho e=\rho\left(Y_{g} e_{g}\left(\frac{Y_{g} \rho}{\alpha_{g}}, \frac{\rho \eta_{g}}{\rho}\right)+Y_{s} e_{s}^{h}\left(\frac{Y_{s} \rho}{1-\alpha_{g}}, \frac{\rho \eta_{s}}{\rho}\right)+Y_{s} e_{s}^{e}\left(\frac{G}{|G|^{1 / 3}}\right)\right)
\end{gathered}
$$

Let us recall a general result proved by Godunov, Friedrichs and Lax : if a system of conservation laws

$$
\mathbf{V}_{t}+\mathbf{G}(\mathbf{V})_{x}=\mathbf{0}
$$


admits an additional conservation law

$$
\varphi(\mathbf{V})_{t}+\psi(\mathbf{V})_{x}=0
$$

where $\varphi(\mathbf{V})$ is a convex function of $\mathbf{V}$, the system (48) is hyperbolic (see Godunov and Romenskii (2003) for detail). In our case this function $\varphi$ is the total volume energy defined from (46) :

$$
E=\rho\left(e+\frac{u^{2}+v^{2}+w^{2}}{2}\right)
$$

and $\mathbf{V}=\left(A^{(1)}, A^{(2)}, A^{(3)}, \rho, \rho Y_{g}, \rho u, \rho v, \rho w, \rho \alpha_{g}, \rho \eta_{s}, \rho \eta_{g}\right)^{T}$. Let us remark that the equations for the volume and mass fractions, and the entropies evolve along contact characteristics. Hence, for hyperbolicity it is sufficient to check the convexity of the energy with respect to a lower number of variables $\hat{\mathbf{V}}=\left(A^{(1)}, A^{(2)}, A^{(3)}, \rho, \rho u, \rho v, \rho w\right)^{T}$. The total energy is in the form :

$$
E=\mathcal{E}^{k}(\rho, \rho u, \rho v, \rho w)+\mathcal{E}^{h}\left(\rho, \alpha_{g}, Y_{g}, \eta_{g}, \eta_{s}\right)+\mathcal{E}_{s}^{e}\left(A^{(1)}, A^{(2)}, A^{(3)}\right)
$$

where the kinetic, hydrodynamic and elastic parts of the volume energy are defined as :

$$
\begin{gathered}
\mathcal{E}^{k}(\rho, \rho u, \rho v, \rho w)=\frac{(\rho u)^{2}+(\rho v)^{2}+(\rho w)^{2}}{2 \rho}, \\
\mathcal{E}^{h}=\mathcal{E}^{h}\left(\rho, \alpha_{g}, Y_{g}, \eta_{g}, \eta_{s}\right)=\rho Y_{g} e_{g}^{h}\left(\rho \frac{Y_{g}}{\alpha_{g}}, \eta_{g}\right)+\rho Y_{s} e_{s}^{h}\left(\rho \frac{1-Y_{g}}{1-\alpha_{g}}, \eta_{s}\right), \\
\mathcal{E}_{s}^{e}\left(A^{(1)}, A^{(2)}, A^{(3)}\right)=\rho Y_{s} e_{s}^{e}(g)=Y_{s} \rho_{0}|G|^{1 / 2} e_{s}^{e}(g) .
\end{gathered}
$$

The energy $E$ is a convex function of $\hat{\mathbf{V}}$, if

$$
\frac{\mathcal{E}^{k}+\mathcal{E}^{h}}{\rho}=\frac{u^{2}+v^{2}+w^{2}}{2}+Y_{g} \varepsilon_{g}^{h}\left(\tau_{g}, \eta_{g}\right)+Y_{g} \varepsilon_{s}^{h}\left(\tau_{s}, \eta_{s}\right)
$$

is convex with respect to $(\tau=1 / \rho, u, v, w)$, and

$$
\mathcal{E}_{s}^{e}=Y_{s} \rho_{0}|G|^{1 / 2} e_{s}^{e}\left(\frac{G}{|G|^{1 / 3}}\right)
$$

is convex with respect to $A^{(\beta)}$. The function $\left(\mathcal{E}^{k}+\mathcal{E}^{h}\right) / \rho$ is convex with respect $(\tau=1 / \rho, u, v, w)$ if the hydrodynamic energies of pure phases $e_{g}^{h}$ and $e_{s}^{h}$ are convex with respect to $\tau_{g}$ and $\tau_{s}$, respectively. This is the case, 
for example, of the stiffened gas equation of state we use for applications. We prove the convexity of $\mathcal{E}_{s}^{e}$ in a special case where

$$
e_{s}^{e}(g)=\frac{\mu_{s}}{4 \rho_{s 0}} \operatorname{tr}\left((g-I)^{2}\right)=\frac{\mu_{s}}{4 \rho_{s 0}}\left(\frac{J_{2}}{|G|^{2 / 3}}-\frac{2 J_{1}}{|G|^{1 / 3}}+3\right) .
$$

Here $\rho_{s 0}$ is the solid reference density. Let us show the convexity of $\mathcal{E}_{s}^{e}$ in the case (49). This energy is in the form :

$$
\begin{aligned}
& \mathcal{E}_{s}^{e}=Y_{s} \frac{\rho_{0}}{\rho_{s 0}} \frac{\mu_{s}}{4}|G|^{1 / 2} \operatorname{tr}\left(\left(\frac{G}{|G|^{1 / 3}}-I\right)^{2}\right) \\
= & Y_{s} \frac{\rho_{0}}{\rho_{s 0}} \frac{\mu_{s}}{4} A^{(1)}\left(\frac{J_{2}}{\left(A^{(1)}\right)^{4 / 3}}-\frac{2 J_{1}}{\left(A^{(1)}\right)^{2 / 3}}+3\right) \\
& =Y_{s} \frac{\rho_{0}}{\rho_{s 0}} \frac{\mu_{s}}{4}\left(\frac{\left(\left(A^{(1)}\right)^{2}+Z^{2}\right)^{2}+2 Z^{2}+2}{\left(A^{(1)}\right)^{1 / 3}}\right. \\
& \left.-2\left(A^{(1)}\right)^{1 / 3}\left(\left(A^{(1)}\right)^{2}+Z^{2}+2\right)+3\right)
\end{aligned}
$$

where

$$
Z^{2}=\left(A^{(2)}\right)^{2}+\left(A^{(3)}\right)^{2}
$$

If the energy $\mathcal{E}_{s}^{e}$ is convex with respect to $A^{(1)}$ and $Z$, and $\partial \mathcal{E}_{s}^{e} / \partial Z>0$, it will be convex with respect to $A^{(1)}, A^{(2)}$ and $A^{(3)}$. Obviously,

$$
\frac{\partial \mathcal{E}_{s}^{e}}{\partial Z}=Y_{s} \frac{\rho_{0}}{\rho_{s 0}} \frac{\mu_{s}}{\left(A^{(1)}\right)^{1 / 3}}\left(Z^{3}+Z\left(\left(A^{(1)}\right)^{2}-\left(A^{(1)}\right)^{2 / 3}+1\right)\right)>0
$$

since for any positive $x$ we have $x^{3}-x+1>0$. Finally, to prove the convexity of $\mathcal{E}_{s}^{e}$ with respect to variables $\left(A^{(1)}, Z\right)$ we have to prove that the corresponding $2 \times 2$ Hessian matrix is positive definite in all domain of parameters $Z>0, A^{(1)}>0$ :

$$
\left(\begin{array}{cc}
\frac{\partial^{2} \mathcal{E}_{s}^{e}}{\partial\left(A^{(1)}\right)^{2}} & \frac{\partial^{2} \mathcal{E}_{s}^{e}}{\partial A^{(1)} \partial Z} \\
\frac{\partial^{2} \mathcal{E}_{s}^{e}}{\partial A^{(1)} \partial Z} & \frac{\partial^{2} \mathcal{E}_{s}^{e}}{\partial Z^{2}}
\end{array}\right)>0
$$

The proof can be done by direct calculations. The symmetrization method assures the hyperbolicity but does not give the characteristic eigenvalues in explicit form. 


\subsection{Applications}

We made a comparison with experimental results on cylindrical gel samples (cylindrical drops of jelly-like materials) impacting a rigid surface (see Figure 2). Jelly-like materials remain elastic even for extreme deformations (between $100 \%$ and $200 \%$ ). This regime was recently studied experimentally by Luu Lia-Hua and Forterre (2009). A cylindrical gel sample (carbopol) impacting a rigid hydrophobic surface, first spreads until some limit size and then a full elastic recoil is observed which may be followed by a complete rebound. We reproduced this quasi-reversible behavior numerically. The separable form of the energy equation was also used with $\mu_{s}=85 \mathrm{~Pa}$. The comparison during the spreading phase at a given time instant is shown in Figure 3.

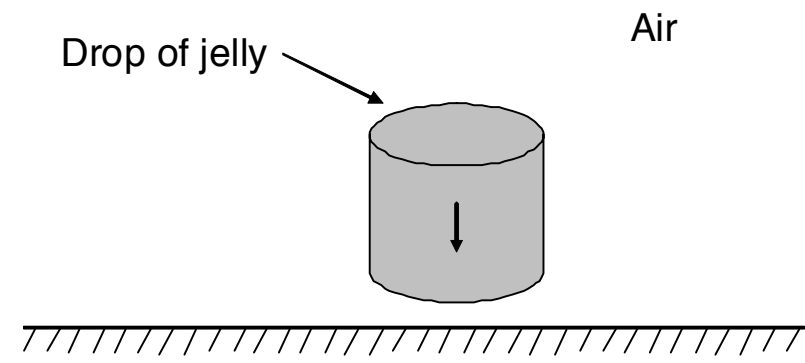

Figure 2. A cylindrical drop of jelly is impacting a rigid surface. 

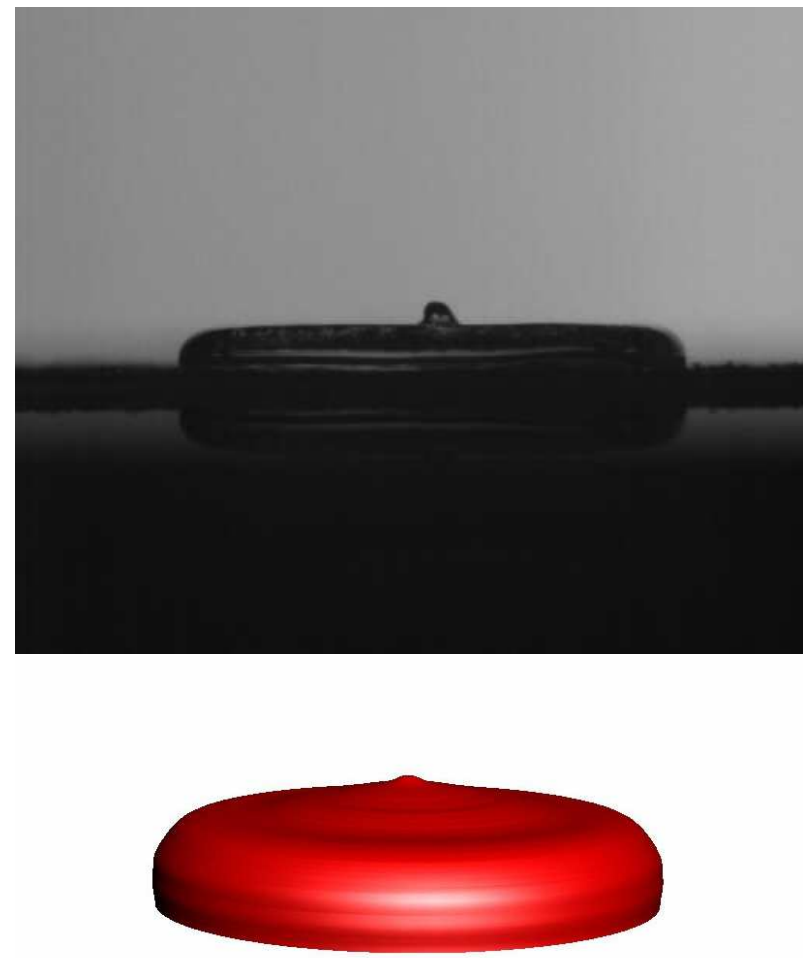

Figure 3. Comparison of the spreading phase of the cylinder impacting a rigid surface. Experiments by L. - H. Luu and Y. Forterre (2009) are shown on the top, computed results are shown on the bottom.

\section{Bibliography}

[1] R. Abgrall (1996) How to prevent pressure oscillations in multicomponent flow calculations : A quasi-conservative approach. J. Comput. Phys. 125, 150-160.

[2] B. Alvarez-Samaniego and D. Lannes (2008) Large time existence for $3 \mathrm{D}$ water waves and asymptotics, Invent. math. v. 171, 485-541.

[3] M. Baer and J. Nunziato (1986) A two-phase mixture theory for the deflagration-to-detonation transition (DDT) in reactive granular materials. Int. J. Multiphase Flows, 12, 861-889.

[4] V. L. Berdichevsky (2009) Variational Principles of Continuum Mechanics, Springer-Verlag Berlin Heidelberg. 
[5] A. D. Drew and S. L. Passman (1996) Theory of multicomponent fluids, Springer-Verlag New York Inc.

[6] N. Favrie, S. L. Gavrilyuk and R. Saurel (2009) Diffuse solid-fluid interface model in cases of extreme deformations, Journal of Computational Physics, 228, 6037-6077.

[7] G. A. El, R. H. J. Grimshaw and N. F. Smyth (2006), Unsteady undular bores in fully nonlinear shallow-water theory, Physics of Fluids 18:027104 (17 pages).

[8] S. L. Gavrilyuk and H. Gouin (1999), A new form of governing equations of fluid arising from Hamilton's principle, Int. J. Eng. Sci. 37:1495-1520.

[9] S. L. Gavrilyuk and V. M. Teshukov (2001), Generalized vorticity for bubbly liquid and dispersive shallow water equations, Continuum Mech. Thermodyn. 13:365-382.

[10] S.L. Gavrilyuk and R. Saurel (2002), Mathematical and Numerical Modeling of Two-Phase Compressible Flows with Microinertia, Journal of Computational Physics, 175, 326-360.

[11] S. L. Gavrilyuk and V. M. Teshukov (2004), Linear stability of parallel inviscid flows of shallow water and bubbly fluid, Stud. Appl. Math. 113:1-29.

[12] S.L. Gavrilyuk, N. Favrie and R. Saurel (2008) Modeling wave dynamics of compressible elastic materials. Journal of Computational Physics, 227, 2941-2969.

[13] S. K. Godunov (1978) Elements of Continuum Mechanics, Nauka, Moscow (in Russian).

[14] S. K. Godunov and E. I. Romenskii (2003) Elements of Continuum Mechanics and Conservation Laws, Kluwer Academic Plenum Publishers, NY.

[15] A. E. Green, N. Laws and P. M. Naghdi (1974), On the Theory of Water Waves, Proceedings of the Royal Society of London A 338: 43-55.

[16] A. E. Green and P. M. Naghdi (1976), A derivation of equations for wave propagation in water of variable depth, J. Fluid Mech. 78:237246.

[17] S. V. Iordansky (1960) On the equations of motion of the liquid containing gas bubbles. Zhurnal Prikladnoj Mekhaniki i Tekhnitheskoj Fiziki N3, 102-111 (in Russian).

[18] M. Ishii and T. Hibiki (2006), Thermo-fluid dynamics of two-phase flow, Springer.

[19] A. K. Kapila, R. Menikoff, J. B. Bdzil, S. F. Son, D. S. Stewart (2001) Two-phase modeling of deflagration-to-detonation transition in granular materials : reduced equations, Phys. Fluids, 13 (10), 3002-3024. 
[20] S. Karni (1994) Multi-component flow calculations by a consistent primitive algorithm, Journal of Computational Physics, 112, 31-43.

[21] B. S. Kogarko (1961) On the model of cavitating liquid. Dokl. AN SSSR 137, 1331-1333 (in Russian).

[22] Y. A. Li (2001) Linear stability of solitary waves of the Green-Naghdi equations, Comm. Pure Appl. Math. 54:501-536.

[23] L.-H. Luu and Y. Forterre (2009) Drop impact of yield-stress fluids, J. Fluid Mechanics, 632, 301-327.

[24] N. Makarenko (1986) A second long-wave approximation in the CauchyPoisson problem, Dynamics of Continuous Media, v. 77, pp. 56-72 (in Russian).

[25] J.-C. Micaelli (1982), Propagation d'ondes dans les écoulements diphasiques à bulles à deux constituants. Etude théorique et expérimentale. Thèse, Université de Grenoble, France.

[26] J. Miles and R. Salmon (1985), Weakly dispersive nonlinear gravity waves, J. Fluid Mech. 157:519-531.

[27] J. N. Plohr and B. J. Plohr (2005) Linearized analysis of RichtmyerMeshkov flow for elastic materials, J. Fluid Mech., 537, 55-89.

[28] R. Saurel and R. Abgrall (2001), A multiphase Godunov method for compressible multifluid and multiphase flows, J. Comput. Physics, 150, 425-467.

[29] R. Saurel, S. L. Gavrilyuk and F. Renaud (2003) A multiphase model with internal degrees of freedom: application to shock-bubble interaction, Journal of Fluid Mechanics, 495, 283-321.

[30] R. Saurel, O. Le Metayer, J. Massoni and S. Gavrilyuk (2007), Shock jump relations for multiphase mixtures with stiff mechanical relaxation, Shock Waves, 16, 209-232.

[31] R. Salmon (1988), Hamiltonian Fluid Mechanics, Ann. Rev. Fluid Mech 20:225-256.

[32] R. Salmon (1998), Lectures on Geophysical Fluid Dynamics, Oxford University Press, New York, Oxford.

[33] C. H. Su and C. S. Gardner (1969), Korteweg-de Vries Equation and Generalizations. III. Derivation of the Korteweg-de Vries Equation and Burgers Equation, J. Math. Phys. 10:536-539.

[34] V. M. Teshukov and S. L. Gavrilyuk (2006), Three-Dimensional Nonlinear Dispersive waves on Shear Flows, Stud. Appl. Math. 116:241-255.

[35] L. van Wijngaarden (1968) On the equations of motion for mixtures of liquid and gas bubbles. J. Fluid Mech. 33, 465-474.

[36] G. B. Whitham (1974), Linear and Nonlinear Waves, John Wiley \& Sons, New York. 